\title{
Planetary Growth with Collisional Fragmentation and Gas Drag
}

\author{
Hiroshi Kobayashi ${ }^{1}$, Hidekazu Tanaka ${ }^{2}$, Alexander V. Krivov ${ }^{1}$, Satoshi Inaba ${ }^{3}$ \\ 1 Astrophysical Institute and University Observatory, Friedrich Schiller University, \\ Schillergaesschen 2-3, 07745, Jena, GERMANY \\ ${ }^{2}$ Institute of Low Temperature Science, Hokkaido University, Kita-Ku Kita 19 Nishi 8, \\ Sapporo 060-0819, JAPAN \\ 3 School of International Liberal Studies, Waseda University, 1-6-1 Nishi-Waseda, \\ Shinjuku-ku, Tokyo 169-8050, JAPAN \\ hkobayas@astro.uni-jena.de
}

Manuscript pages: 53

Figures: 13

Tables: 1 
Running head: Planetary Growth with Fragmentation and Gas Drag

Correspondence Address:

Hiroshi Kobayashi

Astrophysical Institute and University Observatory Friedrich Schiller University Schillergaesschen 2-3, 07745 Jena, GERMANY

Tel: +493641947526

Fax: +493641947532

hkobayas@astro.uni-jena.de

Received __; accepted _ 


\begin{abstract}
As planetary embryos grow, gravitational stirring of planetesimals by embryos strongly enhances random velocities of planetesimals and makes collisions between planetesimals destructive. The resulting fragments are ground down by successive collisions. Eventually the smallest fragments are removed by the inward drift due to gas drag. Therefore, the collisional disruption depletes the planetesimal disk and inhibits embryo growth. We provide analytical formulae for the final masses of planetary embryos, taking into account planetesimal depletion due to collisional disruption. Furthermore, we perform the statistical simulations for embryo growth (which excellently reproduce results of direct $N$-body simulations if disruption is neglected). These analytical formulae are consistent with the outcome of our statistical simulations. Our results indicate that the final embryo mass at several AU in the minimum-mass solar nebula can reach about $\sim 0.1$ Earth mass within $10^{7}$ years. This brings another difficulty in formation of gas giant planets, which requires cores with $\sim 10$ Earth masses for gas accretion. However, if the nebular disk is 10 times more massive than the minimum-mass solar nebula and the initial planetesimal size is larger than 100 $\mathrm{km}$, as suggested by some models of planetesimal formation, the final embryo mass reaches about 10 Earth masses at 3-4 AU. The enhancement of embryos' collisional cross sections by their atmosphere could further increase their final mass to form gas giant planets at 5-10 AU in the solar system.
\end{abstract}

Key Words: Planetary Formation; Planetesimals; Collisional Physics; Origin Solar System; Jovian planets 


\section{INTRODUCTION}

In the standard scenario of planetary formation, terrestrial planets and cores of Jovian planets are formed through the accretion of planetesimals with initial size of $10-100 \mathrm{~km}$

(e.g., Hayashi et al. 1985). This process called planetary accretion has been investigated by statistical simulations (e.g., Wetherill and Stewart 1993; Inaba et al. 2003; Kenyon and Bromley 2004), by $N$-body simulations (e.g., Kokubo and Ida 1996; 2002), and by the hybrid method that combines an $N$-body simulation for large bodies called planetary embryos with a statistical simulation for small bodies (e.g., Bromley and Kenyon 2006; Chambers 2008). Assuming that each collision of bodies leads to a perfect agglomeration, the growth of planetesimals can be accurately computed with $N$-body codes. Inaba et al. (2001) showed that the evolution of mass distribution and velocity dispersion of bodies calculated by $N$-body simulation were reproduced by the statistical simulation applying the collision rate and the velocity-dispersion-evolution rate based on the results of orbital integrations. Therefore, statistical simulations are reliable unless planetary embryos collide with each other after developing a long-term orbital instability.

Planetary embryos initially form through the runaway growth (e.g., Wetherill and Stewart 1989; Kokubo and Ida 1996). The embryos keep their orbital separations and grow through collisions with surrounding planetesimals (Kokubo and Ida 1998). At this stage, planetesimals of almost initial size dominate the total mass of bodies (surface density). When embryos reach about the mass of Mars, the velocity dispersion of planetesimals is increased by the gravitational scattering at the embryos. Accordingly, another growth regime sets in, referred to as oligarchic growth. At this stage, higher velocities of planetesimals cause their collisional fragmentation. After a chain of successive destructive collisions, often called "collision cascade", bodies get smaller and smaller until they are removed by gas drag in protoplanetary disks or by radiation pressure and/or 
Poynting-Robertson drag in debris disks. As a result, collision cascade decreases the surface density, which slows down the growth of planetary embryos (Inaba et al. 2003; Kenyon and Bromley 2008).

The planetary core (embryo) exceeding the critical core mass as large as 10 Earth masses can no longer retain a hydrostatic envelope, resulting in the gas accretion and formation of the gas giant planets (e.g., Mizuno et al. 1980; Bodenheimer \& Pollack 1986; Pollack et al. 1996; Ikoma et al. 2001). However, since the embryo growth is hindered by the planetesimal depletion in collision cascade, the final embryo can hardly grow beyond the Mars mass and thus cannot form a gas giant (Kobayashi and Tanaka 2010).

However, it is possible that fragmentation inhibits the embryo growth to a lesser extent than ascertained before. Collisional fragments orbiting a central star drift inward by gas drag. The drift time shortens as bodies become smaller by collision cascade, until at a certain size their motion gets coupled with gas motion. Such coupled bodies have lower drift velocity and thus can survive around planetary embryos for longer time. Kenyon and Bromley (2009) suggested that accretion of those coupled bodies by planetary embryos may promote further growth of the latter. In their simulations, they assumed that the coupled bodies no longer experience an inward drift. However, the drift, although at a reduced rate, is important to determine the embryo mass gain due to the accretion of coupled bodies.

This paper investigates the embryo growth taking into account the accretion of fragments resulting from the collision cascade before their removal by gas drag. To this end, we perform an analytic study that extends the model of Kobayashi and Tanaka (2010) by including the removal by gas drag, as well as statistical simulations. In the numerical treatment, we do not neglect the drift of coupled bodies by gas drag. Instead, we take into account that the gas drag law changes for small bodies in the both analytical and numerical procedure (e.g., Adachi et al. 1976). The goal is to find out to what extent the collisional 
fragmentation combined with gas drag would affect the embryo growth and whether an embryo can reach the critical core mass.

We develop the analytic theory and derive the final embryo mass with fragmentation and gas drag in Section 2. In Section 3, we check the formulae for the final mass against the statistical numerical simulations. Section 4 contains a summary and a discussion of our results.

\section{THEORETICAL MODEL}

In this section, we summarize the oligarchic growth of planetary embryos and the surface density decline resulting from fragmentation. Then we derive the general formulae for the final mass through the oligarchic growth with fragmentation.

\subsection{DISK AND FRAGMENTATION MODEL}

We introduce a power-law disk model for the surface mass density of solids and gas. The solid surface mass density is taken to be

$$
\Sigma_{\mathrm{s}, 0}=f_{\mathrm{ice}} \Sigma_{1}\left(\frac{a}{1 \mathrm{AU}}\right)^{-q} \mathrm{~g} \mathrm{~cm}^{-2}
$$

where $a$ is the distance from a central star, $\Sigma_{1}$ is the reference surface density at $1 \mathrm{AU}$, $q$ is the power-law index of the radial distribution, and $f_{\text {ice }}$ is the factor that represents the increase of solid density by ice condensation beyond the snow line $a_{\text {ice }}$ at which the temperature equals the ice condensation temperature $\simeq 170 \mathrm{~K}$. We set the gas surface density to

$$
\Sigma_{\text {gas }, 0}=f_{\text {gas }} \Sigma_{1}\left(\frac{a}{1 \mathrm{AU}}\right)^{-q} \mathrm{~g} \mathrm{~cm}^{-2}
$$


where $f_{\text {gas }}$ is the gas-dust ratio. In the nominal case, we adopt $f_{\text {gas }}=240$ (Hayashi 1981). If the disk is optically thin, the gas temperature is given by

$$
T=280\left(\frac{a}{1 \mathrm{AU}}\right)^{-1 / 2}\left(\frac{L_{*}}{L_{\odot}}\right)^{1 / 4} \mathrm{~K},
$$

where $L_{*}$ and $L_{\odot}$ are luminosities of the central star and the sun, respectively. For $L_{*}=L_{\odot}$, this yields $a_{\text {ice }}=2.7 \mathrm{AU}$. In the minimum-mass solar nebula (MMSN) model, $\Sigma_{1}=7 \mathrm{~g} / \mathrm{cm}^{2}$, $q=3 / 2$ and $f_{\text {ice }}=1\left(a<a_{\text {ice }}\right)$ and $4.2\left(a>a_{\text {ice }}\right)$. However, since a large amount of small dust is present even after completion of planetesimal formation, the disk is expected to be optically thick. This would make the temperature lower and its radial profile different (e.g., Kusaka et al. 1970). However, these effects would not drastically influence the embryo growth except for the location of the snow line.

We take the fragmentation model described by Kobayashi and Tanaka (2010). Assuming that the fragmentation is scaled by the energy, the total ejecta mass $m_{\mathrm{e}}$ produced by one collision between $m_{1}$ and $m_{2}$ is given by

$$
\frac{m_{\mathrm{e}}}{m_{1}+m_{2}}=\frac{\phi}{1+\phi} .
$$

Here the scaled impact energy $\phi$ is given by $m_{1} m_{2} v^{2} / 2\left(m_{1}+m_{2}\right)^{2} Q_{\mathrm{D}}^{*}$, where $v$ is the collisional velocity between $m_{1}$ and $m_{2}$ and $Q_{\mathrm{D}}^{*}$ is the critical specific impact energy needed to disrupt the colliding bodies and eject $50 \%$ of their mass $\left(m_{\mathrm{e}}=\left(m_{1}+m_{2}\right) / 2\right)$. The value of $Q_{\mathrm{D}}^{*}$ is given by the larger of the two colliders $\left(m_{1}\right.$ and $\left.m_{2}\right)$. Note that, since Kobayashi and Tanaka (2010) separately determine the ejecta mass from $m_{1}$ and $m_{2}$ in their analysis, Eq. (44) is different from their definition.

The energy threshold is given by

$$
Q_{\mathrm{D}}^{*}=Q_{0 \mathrm{~s}}\left(\frac{r}{1 \mathrm{~cm}}\right)^{\beta_{\mathrm{s}}}+Q_{0 \mathrm{~g}} \rho\left(\frac{r}{1 \mathrm{~cm}}\right)^{\beta_{\mathrm{g}}}+C_{\mathrm{gg}} \frac{2 G m}{r},
$$

where $r$ and $m$ are the radius and mass of a body and $\rho$ is its density. Benz and Ashpaug (1999) provide $Q_{0 \mathrm{~s}}, \beta_{\mathrm{s}}, Q_{0 \mathrm{~g}}$, and $\beta_{\mathrm{g}}$ for $r=1-10^{7} \mathrm{~cm}$ from the hydrodynamical simulation of 
the collisional dispersion. The first term in the right-hand side of Eq. (5) controls $Q_{\mathrm{D}}^{*}$ for $r \lesssim 10^{4}-10^{5} \mathrm{~cm}$ and the second term describes $Q_{\mathrm{D}}^{*}$ of the larger bodies. For $r \gtrsim 10^{7} \mathrm{~cm}$, $Q_{\mathrm{D}}^{*}$ is purely determined by the gravitational binding energy, being independent of material properties. The collisional simulation for the gravitational aggregates yields $C_{\mathrm{gg}} \simeq 10$ (Stewart and Leinhardt 2009).

\subsection{ISOLATION MASS}

Planetary embryos no longer grow after they have accreted all planetesimals within their feeding zones. The width of a feeding zone is equal to the orbital separation of the embryos, $\tilde{b}\left(2 M / 3 M_{*}\right)^{1 / 3} a$, where $M$ is the embryo mass, $M_{*}$ is the mass of central star, $\tilde{b} \simeq 10$ is a factor (Kokubo and Ida 2002). Therefore, the maximum mass or "isolation mass" satisfy $M_{\text {iso }}=2 \pi a^{2}\left(2 M_{\text {iso }} / 3 M_{*}\right)^{1 / 3} \tilde{b} \Sigma_{\mathrm{s}}$. It can be expressed as

$$
M_{\text {iso }}=2.8\left(\frac{\tilde{b}}{10}\right)^{3 / 2}\left(\frac{\Sigma_{\mathrm{s}, 0}}{2.7 \mathrm{~g} / \mathrm{cm}^{2}}\right)^{3 / 2}\left(\frac{a}{5 \mathrm{AU}}\right)^{3}\left(\frac{M_{*}}{M_{\odot}}\right)^{-1 / 2} M_{\oplus},
$$

where $M_{\oplus}$ is the Earth mass and $M_{\odot}$ is the solar mass. The planetary embryo mass approaches the isolation mass if fragmentation is ignored (Kokubo and Ida 2000; 2002). However, if fragmentation is included, the final embryo mass is expected to be smaller.

\subsection{PLANETARY GROWTH}

\subsubsection{GROWTH WITHOUT ACCRETION OF FRAGMENTS}

At the runaway stage of planetary growth, the larger planetesimals grow faster than smaller ones. The solid surface density at the runaway growth stage is determined by relatively small planetesimals. At the later, oligarchic stage, planetary embryos become massive, start to gravitationally stir up the planetesimals and induce their collisional 
disruption. The resulting fragments are quickly removed by the inward drift due to gas drag. Thus the fragmentation would reduce the surface density and hence the final embryo mass. Here, taking into account the depletion of planetesimals due to the fragmentation, we estimate the final embryo mass. We neglect the accretion of fragments onto the embryos, whereas the accretion of fragments will be considered in Section 2.3.2.

At the oligarchic stage, planetary embryos are distributed uniformly due to their mutual gravitational interaction. Since they cannot collide with each other due to their large orbital separation, they grow slowly. If a planetary embryo with mass $M$ collides with planetesimals of mass $m$, its growth rate is given by

$$
\frac{d M}{d t}=\int d m m n_{\mathrm{s}} a^{2} h_{m, M}^{2}\left\langle P_{\mathrm{col}}\right\rangle \Omega_{\mathrm{K}},
$$

where $n_{\mathrm{s}} d m$ is the surface number density of planetesimals with mass in the range of $[m, m+d m], \Omega_{\mathrm{K}}$ is the Kepler angular velocity, $h_{m, M}=\left[(m+M) / 3 M_{*}\right]^{1 / 3}$ is the dimensionless reduced Hill radius between $m$ and $M$, and $\left\langle P_{\text {col }}\right\rangle$ is the dimensionless collision rate. We assume $m \ll M$, and Eq. (17) reduces to

$$
\frac{d M}{d t}=C_{\mathrm{acc}} \Sigma_{\mathrm{s}} a^{2} h_{\mathrm{M}}^{2}\left\langle P_{\mathrm{col}}\right\rangle \Omega_{\mathrm{K}}
$$

where $\Sigma_{\mathrm{s}}$ is the surface density of planetesimals, $h_{M}=\left(M / 3 M_{*}\right)^{1 / 3}$, and $C_{\text {acc }}$ is the correction factor on the order of unity. Because the eccentricity dispersion $e^{*}$ and inclination dispersion $i^{*}$ of planetesimals are much larger than $h_{M}$ for kilometer-sized or larger planetesimals, the dimensionless collision rate $\left\langle P_{\text {col }}\right\rangle$ is given by (e.g., Inaba et al. 2001)

$$
\left\langle P_{\mathrm{col}}\right\rangle=\frac{C_{\mathrm{col}} \tilde{R} h_{M}^{2}}{e^{* 2}}
$$

where $C_{\text {col }} \simeq 36$ for $e^{*}=2 i^{*}$. Here, $\tilde{R}=\left(9 M_{*} / 4 \pi \rho\right)^{1 / 3} / a$ is the embryo radius scaled by $h_{M} a$ and independent of the embryo mass. In this paper, we do not take into account the enhancement of $\left\langle P_{\text {col }}\right\rangle$ due to atmosphere which an embryo is expected to acquire. 
Such an enhancement may be efficient for $M \gtrsim 0.1 M_{\oplus}$ (Inaba and Ikoma 2003). Note that embryos grow not only through collisions with swarm planetesimals but also with neighboring embryos because their separation scaled by the Hill radius decreases by their growth. Furthermore, taking into account mass distribution of planetesimals, the actual accretion rate becomes larger. In this paper, we apply $C_{\mathrm{acc}} \simeq 1.5$.

Since $d M / d t \propto e^{*-2}$, we need to describe evolution of $e^{*}$. Two mechanisms that affect $e^{*}$ are viscous stirring and damping by gas. The viscous stirring of planetary embryos raises the random velocities of planetesimals. The stirring rate of $e^{* 2}$ is given by

$$
\left(\frac{d e^{* 2}}{d t}\right)_{\mathrm{VS}}=n_{M} a^{2} h_{M}^{4}\left\langle P_{\mathrm{VS}}\right\rangle \Omega_{\mathrm{K}} .
$$

Since embryos are uniformly distributed with a separation $2^{1 / 3} h_{\mathrm{M}} a \tilde{b}$, their surface number density $n_{M}$ is given by

$$
n_{M}=\frac{1}{2^{4 / 3} \pi \tilde{b} h_{M} a^{2}} .
$$

For $e^{*}=2 i^{*} \gg h_{M}$, the dimensionless stirring rate $\left\langle P_{\mathrm{VS}}\right\rangle$ is given by (Ohtsuki et al. 2002)

$$
\left\langle P_{\mathrm{VS}}\right\rangle=\frac{C_{\mathrm{VS}} h_{M}^{2}}{e^{* 2}} \ln \left(\Lambda^{2}+1\right)
$$

where $C_{\mathrm{VS}} \simeq 40$. Although $\ln \left(\Lambda^{2}+1\right)$ weakly depends on $e^{*}$, we assume $\ln \left(\Lambda^{2}+1\right) \simeq 3$ for this analysis. On the other hand, $e^{*}$ is damped by gas drag and the damping rate is given by

$$
\left(\frac{d e^{* 2}}{d t}\right)_{\text {gas }}=-\frac{C_{\text {gas }}}{\tau} e^{* 3},
$$

where $C_{\text {gas }} \simeq 2.1$ (e.g., Inaba et al. 2001) and

$$
\tau=\frac{m}{\pi r^{2} C_{\mathrm{D}} \rho_{\mathrm{gas}} v_{\mathrm{K}}}
$$

Here, $\rho_{\text {gas }}$ is the gas density in the midplane, $v_{\mathrm{K}}$ is the Keplerian velocity, and $C_{\mathrm{D}}$ is the dimensionless gas drag coefficient. The latter is defined as a factor that appears in the expression for the gas drag force acting on a planetesimal with radius $r: C_{\mathrm{D}} \pi r^{2} \rho_{\text {gas }} u^{2} / 2$ with 
$u$ being the relative velocity between the planetesimal and gas. For kilometer-sized or larger planetesimals, $C_{\mathrm{D}}$ is a constant (Adachi et al. 1976). We assume that $e^{*}$ is determined by the equilibrium between the stirring and the damping (Eqs. (10) and (13)). Equating the stirring and damping rates, we obtain

$$
\frac{e^{*}}{h_{M}}=\left[\frac{C_{\mathrm{VS}} \ln \left(\Lambda^{2}+1\right) \Omega_{\mathrm{K}} \tau}{2^{4 / 3} \pi \tilde{b} C_{\text {gas }}}\right]^{1 / 5} .
$$

We can estimate $e^{*} \sim\left(\tau \Omega_{\mathrm{K}}\right)^{1 / 5} h_{M}$. Since $\tau \Omega_{\mathrm{K}} \gg 1$ for the planetesimals (Adachi et al. 1976), our assumption of $e^{*} \gg h_{M}$ for the derivation is valid. Inserting Eqs. (9) and (15) to Eq. (8), the growth rate is found to be

$$
\frac{d M}{d t}=C_{\mathrm{acc}} \Sigma_{\mathrm{s}} a^{2} C_{\mathrm{col}} \tilde{R} \Omega_{\mathrm{K}}\left[\frac{2^{4 / 3} \pi \tilde{b} C_{\mathrm{gas}}}{C_{\mathrm{VS}} \ln \left(\Lambda^{2}+1\right) \Omega_{\mathrm{K}} \tau}\right]^{2 / 5} h_{M}^{2} .
$$

The time evolution of a planetary embryo in the oligarchic growth mode, neglecting fragmentation, is summarized in Appendix A.

As embryos grow, the random velocity of planetesimals increases, making collisions between planetesimals destructive. Fragments produced by the collisions get smaller and smaller by successive collisions (collision cascade) until the smallest ones are brought inward by gas drag and are lost to the central star. Planetesimals with mass $m$ dominate the surface density $\Sigma_{\mathrm{s}}$ during the oligarchic growth. Thus, the collision cascade induced by embryo growth reduces the surface density. The evolution of $\Sigma_{\mathrm{s}}$ due to a collision cascade is given by (see Kobayashi and Tanaka 2010; Appendix B for a derivation)

$$
\frac{d \Sigma_{\mathrm{s}}}{d t}=-\frac{\Sigma_{\mathrm{s}}^{2}(2-\alpha)^{2} h_{0} \Omega_{\mathrm{K}}}{m^{1 / 3}}\left(\frac{v(m)^{2}}{2 Q_{\mathrm{D}}^{*}(m)}\right)^{\alpha-1} s_{123}(\alpha),
$$

where $h_{0}=1.1 \rho^{-2 / 3}$ and

$$
s_{123}(\alpha)=\int_{0}^{\infty} d \phi \frac{\phi^{-\alpha}}{1+\phi}\left\{\left[-\ln \frac{\epsilon \phi}{(1+\phi)^{2}}+\frac{1}{2-b}\right] \phi+\ln (1+\phi)\right\},
$$

where $b$ is the power-law exponent of the mass distribution of ejecta yielded by one collision between $m_{1}$ and $m_{2}$ and $\epsilon$ is a factor in the expression for the mass of the largest ejecta: 
$m_{\mathrm{L}}=\epsilon\left(m_{1}+m_{2}\right) \phi /(1+\phi)^{2}$ with a use of the scaled impact energy $\phi$. For a collision cascade, the mass distribution exponent $\alpha$ of fragments is given by $\alpha=(11+3 p) /(6+3 p)$ for $v(m)^{2} / Q_{\mathrm{D}}^{*}(m) \propto m^{-p}$ and $s_{123}$ is insensitive to constants $\epsilon<1$ and $b=1-2$ (Kobayashi and Tanaka 2010). We set $b=5 / 3$ and $\epsilon=0.2$.

Dividing Eq. (16) by Eq. (17) and integrating, we obtain the final embryo mass,

$$
\begin{aligned}
M_{\mathrm{c}}= & \left(\frac{2 \alpha-1}{3}\right)^{3 /(2 \alpha-1)}\left(\frac{a^{2} C_{\mathrm{acc}} C_{\mathrm{col}} \tilde{R} m^{1 / 3}}{(2-\alpha)^{2} h_{0} s_{123}}\right)^{3 /(2 \alpha-1)}\left(\frac{v_{\mathrm{K}}^{2}}{2 Q_{\mathrm{D}}^{*}}\right)^{3(1-\alpha) /(2 \alpha-1)} \\
& \times\left(3 M_{*}\right)^{-(4-2 \alpha) /(2 \alpha-1)}\left[\ln \left(\frac{\Sigma_{\mathrm{s}, 0}}{\Sigma_{\mathrm{s}}}\right)\right]^{3 /(2 \alpha-1)}\left[\frac{C_{\mathrm{VS}} \ln \left(\Lambda^{2}+1\right) \Omega_{\mathrm{K}} \tau}{2^{4 / 3} \pi \tilde{b} C_{\mathrm{gas}}}\right]^{-6 \alpha / 5(2 \alpha-1)},
\end{aligned}
$$

where $v=e^{*} v_{\mathrm{K}}$. This equation is valid unless the surface density is much smaller than that of embryos, $\Sigma_{\mathrm{s}} \ll M_{\mathrm{c}} n_{M}$. Therefore, an estimate of $\Sigma_{\mathrm{s}}$ for the final embryo mass is given by

$$
\frac{\Sigma_{\mathrm{s}}}{\Sigma_{\mathrm{s}, 0}}=\frac{C_{\Sigma_{\mathrm{s}}} M_{\mathrm{c}}}{2^{4 / 3} \pi \tilde{b} a^{2} h_{M_{\mathrm{c}}} \Sigma_{\mathrm{s}, 0}}=C_{\Sigma_{\mathrm{s}}}\left(\frac{M_{\mathrm{c}}}{M_{\mathrm{iso}}}\right)^{2 / 3},
$$

where $C_{\Sigma_{\mathrm{s}}} \ll 1$ is a constant. Generally, $M_{\mathrm{c}}$ should be smaller than $M_{\text {iso }}$.

We assume $Q_{\mathrm{D}}^{*}=Q_{0 \mathrm{~g}} \rho r^{\beta_{\mathrm{g}}}$ for the gravity regime and thus $\alpha=\left[11+3\left(\beta_{\mathrm{g}}-2 / 15\right)\right] /[6+$ $3(\beta-2 / 15)]$. For the minimum-mass solar nebula $\left(\Sigma_{0}=7 \mathrm{~g} / \mathrm{cm}^{2}\right.$ and $\left.q=3 / 2\right)$, Eq. (19) can be re-written as

$$
\begin{aligned}
& M_{\mathrm{c}}=0.10\left(\frac{\ln \left(\Sigma_{\mathrm{s}, 0} / \Sigma_{\mathrm{s}}\right)}{4.5}\right)^{1.21}\left(\frac{a}{5 \mathrm{AU}}\right)^{0.63}\left(\frac{m}{4.2 \times 10^{20} \mathrm{~g}}\right)^{0.48} \\
& \times\left(\frac{M_{*}}{M_{\odot}}\right)^{-0.28}\left(\frac{Q_{0 \mathrm{~g}}}{2.1 \mathrm{erg} \mathrm{c \textrm {cm } ^ { 3 } / \mathrm { g } ^ { 2 }}}\right)^{0.89}\left(\frac{\rho}{1 \mathrm{~g} / \mathrm{cm}^{3}}\right)^{1.85} M_{\oplus}
\end{aligned}
$$

where $\beta_{\mathrm{g}}=1.19$ for ice. Here, we estimate $\ln \left(\Sigma_{s} / \Sigma_{\mathrm{s}, 0}\right) \simeq 4.5$ from Eq. (20) with the use of $C_{\Sigma_{\mathrm{s}}}=0.1$ for $M_{\mathrm{c}}=0.10 M_{\oplus}$ and $M_{\text {iso }}=2.8 M_{\oplus}$. With fragmentation, the final embryo mass given by Eq. (21) becomes much smaller than the isolation mass, Eq. (6) $)$. As we will show in Section 3 with the aid of statistical simulations, the planetesimal mass $m$ is about $100 m_{0}$, where $m_{0}$ is the initial planetesimal mass. We assume $m=100 m_{0}$ and will compare $M_{\mathrm{c}}$ with the embryo mass obtained through the statistical simulation. 


\subsubsection{GROWTH WITH ACCRETION OF FRAGMENTS}

For the steady-state mass distribution of collision cascade (see Appendix A), the surface density of small fragments is much lower than that of planetesimals and the accretion of fragments is insignificant for embryo growth. The collision cascade may terminate at a certain mass where destructive collisions no longer occur due to low collisional velocities damped by a strong gas drag. The steady-state mass distribution is achieved if fragments at the low-mass end of the cascade are quickly removed by the gas drag. However, when the $\Sigma_{\mathrm{s}}$-decay time resulting from the collision cascade is shorter than the removal time of the fragments by gas drag, the termination of the collision cascade yields a large amount of fragments at the low-mass end of the cascade and would dominate the solid surface density. Here, we examine the termination of the collision cascade and estimate the embryo growth through accretion of such fragments.

For small fragments, the $e^{*}$-damping rate resulting from the gas drag is given by (Adachi et al. 1976)

$$
\left(\frac{d e^{*}}{d t}\right)_{\mathrm{gas}, \mathrm{f}}=-\frac{e^{*} \eta}{\tau},
$$

where $\eta=\left(v_{\mathrm{K}}-v_{\text {gas }}\right) / v_{\mathrm{K}}$ is the deviation of the gas rotation velocity $v_{\text {gas }}$ from the Keplerian velocity. For $q=3 / 2, \eta=1.8 \times 10^{-3}(a / 1 \mathrm{AU})^{1 / 2}$. For small fragments, $C_{\mathrm{D}}$ depends on $u$ and $e^{*}$ becomes much smaller than $\eta$ by the gas drag. Hence, the damping rate given by Eq. (22) is different from Eq. (13). Since the gas drag substantially damps $e^{*}$ of small fragments, $\left\langle P_{\mathrm{VS}}\right\rangle=73$ is independent of $e^{*}$ for $e^{*} \ll h_{M}$. Thus, the equilibrium condition between the stirring (Eqs. (10)-(11) ) and the damping (Eq. (22)) gives

$$
e^{* 2}=\frac{h_{M}^{3}\left\langle P_{\mathrm{VS}}\right\rangle \tau \Omega_{\mathrm{K}}}{2^{4 / 3} \pi \tilde{b} \eta} .
$$

Smaller fragments have low $e^{*}$ because of low $\tau$.

The collision cascade will no longer operate for such low $e^{*}$. The collisional energy 
between bodies of mass $m$ is estimated to be $m e^{* 2} v_{\mathrm{K}}^{2} / 4$ and is much smaller than energy threshold $2 m Q_{\mathrm{D}}^{*}$ for $m_{\mathrm{e}}=m$ at the low-mass end of the cascade. Therefore, at the low-mass end

$$
e^{* 2} v_{\mathrm{K}}^{2}=C_{\mathrm{L}} Q_{\mathrm{D}}^{*}
$$

where $C_{\mathrm{L}}$ is a constant. As we will show, the surface density of fragments becomes higher at the low-mass end of the collision cascade. Our simulation yields $C_{\mathrm{L}} \simeq 1$ at the low-mass end.

The surface density of the fragments decreases as a result of the radial drift by gas drag. From Eqs. (23) and (24), we estimate the scaled stopping time $\tilde{\tau}_{\text {stop }}$ of the fragments at the low-mass end of the cascade to be

$$
\begin{aligned}
\tilde{\tau}_{\text {stop }} & =\frac{\tau \Omega_{\mathrm{K}}}{\eta}=\frac{2^{4 / 3} \pi \tilde{b}}{h_{M}^{3}\left\langle P_{\mathrm{VS}}\right\rangle} \frac{C_{\mathrm{L}} Q_{\mathrm{D}}^{*}}{v_{\mathrm{K}}^{2}} \\
& \simeq 19 C_{\mathrm{L}}\left(\frac{a}{5 \mathrm{AU}}\right)\left(\frac{M}{0.1 M_{\oplus}}\right)^{-1}\left(\frac{Q_{\mathrm{D}}^{*}}{3.1 \times 10^{6} \mathrm{erg} / \mathrm{g}}\right)
\end{aligned}
$$

where $Q_{\mathrm{D}}^{*} \simeq 3.1 \times 10^{6} \mathrm{erg} / \mathrm{g}$ for ice bodies with $r=10 \mathrm{~m}$ and we will derive $M \sim 0.1 M_{\oplus}$ in the following analysis. Since $\tilde{\tau}_{\text {stop }} \gg 1$, the coupling between the fragments and gas is weak. Therefore, the drift velocity of fragments is given by $2 \eta^{2} a / \tau$ (Adachi et al. 1976). Since $\tau \propto a^{3 / 4}$ for the fragments and $\Sigma_{\mathrm{s}} \propto a^{-q}$, the $\Sigma_{\mathrm{s}}$-depletion rate is given by $d \Sigma_{\mathrm{S}} / d t=-2(9 / 4-q) \eta^{2} \Sigma_{\mathrm{s}} / \tau$. Eliminating $\tau$ of fragments at the low-mass end of the cascade by Eq. (25), we get

$$
\frac{d \Sigma_{\mathrm{s}}}{d t}=-\left(\frac{9}{4}-q\right) \frac{\Sigma_{\mathrm{s}} h_{M}^{3}\left\langle P_{\mathrm{VS}}\right\rangle \Omega_{\mathrm{K}} \eta v_{\mathrm{K}}^{2}}{2^{1 / 3} C_{\mathrm{L}} \pi \tilde{b} Q_{\mathrm{D}}^{*}} .
$$

Since $\left\langle P_{\text {col }}\right\rangle \simeq 11.3 \sqrt{\tilde{R}}$ is independent of $M$ for $e^{*} \ll h_{M}$, integration of Eq. (8) divided by Eq. (27) gives another formula for the final embryo mass,

$$
M_{\mathrm{f}}=\left[\frac{2^{7 / 3} C_{\mathrm{L}} \tilde{b} \pi C_{\mathrm{acc}} a^{2}\left\langle P_{\mathrm{col}}\right\rangle\left(3 M_{*}\right)^{1 / 3}}{3(9 / 4-q) \eta P_{\mathrm{VS}}} \frac{Q_{\mathrm{D}}^{*}}{v_{\mathrm{K}}^{2}}\left(\Sigma_{\mathrm{s}, 0}-\Sigma_{\mathrm{s}}\right)\right]^{3 / 4},
$$


where we set $M=0$ at $\Sigma_{\mathrm{s}}=\Sigma_{\mathrm{s}, 0}$. We recall that $\left\langle P_{\mathrm{VS}}\right\rangle=73$ and $\left\langle P_{\mathrm{col}}\right\rangle=11.3 \sqrt{\tilde{R}}$ in Eq. (28) are independent of $M_{\mathrm{f}}$ for $e^{*} \ll h_{M}$. In addition, since $Q_{\mathrm{D}}^{*}$ for the strength regime

is almost independent of the fragment mass $m$, we neglected the mass dependence of $Q_{\mathrm{D}}^{*}$ for the derivation of Eq. (28). Assuming $\Sigma_{\mathrm{s}, 0} \gg \Sigma_{\mathrm{s}}$ and $q=3 / 2$, we obtain

$$
\begin{aligned}
M_{\mathrm{f}}= & 0.14\left(\frac{\tilde{b}}{10}\right)^{3 / 4}\left(\frac{a}{5 \mathrm{AU}}\right)^{3 / 2}\left(\frac{M_{*}}{M_{\odot}}\right)^{3 / 8} \\
& \times\left(\frac{\rho}{1 \mathrm{~g} / \mathrm{cm}^{3}}\right)^{-1 / 8}\left(\frac{\Sigma_{\mathrm{s}, 0}}{2.7 \mathrm{~g} / \mathrm{cm}^{2}}\right)^{3 / 4}\left(\frac{Q_{\mathrm{D}}^{*}}{3.1 \times 10^{6} \mathrm{erg} / \mathrm{g}}\right)^{3 / 4} M_{\oplus}
\end{aligned}
$$

The final mass given by Eq. (29) is also much smaller than the isolation mass, Eq. (6) . Furthermore, $M_{\mathrm{f}}$ is comparable to $M_{\mathrm{c}}$ given by Eq. (21). When planetesimals have a large $Q_{\mathrm{D}}^{*}$ because of a rigid material (high $Q_{0 \mathrm{~g}}$ ) and/or a large size, $M_{\mathrm{c}}$ is larger than $M_{\mathrm{f}}$. In the opposite case, $M_{\mathrm{f}}$ exceeds $M_{\mathrm{c}}$. The final mass is supposed to be the larger of the two, $M_{\mathrm{c}}$ and $M_{\mathrm{f}}$. We apply $Q_{\mathrm{D}}^{*}$ for $r=10 \mathrm{~m}$ and will compare $M_{\mathrm{f}}$ with the embryo mass resulting from the statistical simulation.

\section{NUMERICAL SIMULATIONS}

\subsection{METHOD}

Many authors attacked the problem of the planetary growth from planetesimals to embryos by $N$-body simulations (e.g., Kokubo and Ida 1996; 2002), the statistical method (Wetherill and Stewart 1993; Kenyon and Bromley 2004), and the hybrid method (Kenyon and Bromley 2008, Chambers 2008). We apply the statistical method developed by Inaba et al. (1999, 2001), which we briefly explain now. The mass distribution of bodies in orbit around a central star evolves through mutual collisions, taking into account gravitational focusing of colliding bodies (see Inaba et al. 2001 for detailed expressions). Along with the mass evolution, velocity dispersion changes by gravitational perturbations, collisional 
damping, and gas drag. Equations for the mass distribution of bodies are integrated simultaneously with equations for their velocity distribution.

The time evolution of the differential surface number density $n_{\mathrm{s}}(m)$ at a distance $a$ is given by

$$
\begin{aligned}
\frac{\partial m n_{\mathrm{s}}(m, a)}{\partial t}=\frac{m}{2} \Omega_{\mathrm{K}} & \int_{0}^{m} d m_{1} \int_{m-m_{1}-m_{\mathrm{e}}}^{\infty} d m_{2} \\
& \times\left(h_{m_{1}, m_{2}} a\right)^{2} n_{\mathrm{s}}\left(m_{1}, a\right) n_{\mathrm{s}}\left(m_{2}, a\right)\left\langle P_{\mathrm{col}}\right\rangle \\
& \times \delta\left(m-m_{1}-m_{2}+m_{\mathrm{e}}\right) \\
& -\Omega_{\mathrm{K}} m n_{\mathrm{s}}(m) \int_{0}^{\infty} d m_{2}\left(h_{m, m_{2}} a\right)^{2} n_{\mathrm{s}}\left(m_{2}, a\right)\left\langle P_{\mathrm{col}}\right\rangle \\
+ & \frac{\partial}{\partial m} \Omega_{\mathrm{K}} \int_{m}^{\infty} d m_{1} \int_{0}^{m_{1}} d m_{2}\left(m_{1}+m_{2}\right) f\left(m, m_{1}, m_{2}\right) \\
& \times n_{\mathrm{s}}\left(m_{1}, a\right) n_{\mathrm{s}}\left(m_{2}, a\right)\left(h_{m_{1}, m_{2}} a\right)^{2}\left\langle P_{\mathrm{col}}\right\rangle \\
& -\frac{1}{a} \frac{\partial}{\partial a}\left[a m n_{\mathrm{s}}(m, a) v_{\mathrm{drift}}(m, a)\right],
\end{aligned}
$$

where $\delta(x)$ is the delta function, $\left(m_{1}+m_{2}\right) f\left(m, m_{1}, m_{2}\right)$ is the mass of fragments less than $m$ produced by a collision between $m_{1}$ and $m_{2}$, and $m_{\mathrm{e}}$ is the total mass of the fragments (given by Eq. (4) in our fragmentation model). We apply the dimensionless collisional rate $\left\langle P_{\text {col }}\right\rangle$ which Inaba et al. (2001) provide as a function of $m_{1}, m_{2}$, and $e^{*}$ and $i^{*}$ by compiling previous studies. Equation (30) describes the mass transport in the two-dimensional space composed of mass and distance (radial direction). The first and second terms in the right-hand side of Eq. (30) represent the mass transport along the mass coordinate caused by coagulation and the third term does that due to fragmentation. The fourth term describes the mass transport along the radial coordinate due to the drift of bodies. We calculate the transport on a grid of mass and radial bins.

Assuming a power-law mass distribution of fragments, $\left(m_{1}+m_{2}\right) f\left(m, m_{1}, m_{2}\right)$ is given 
by

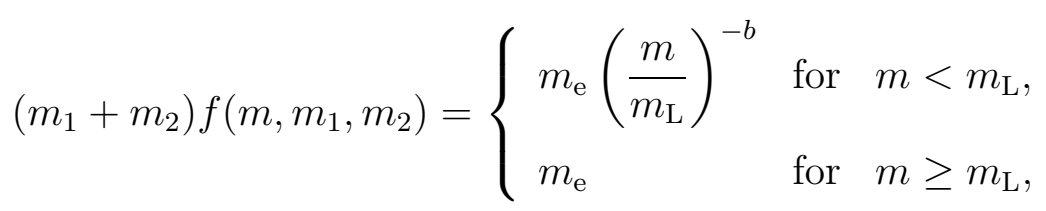

where we recall the mass of the largest ejecta $m_{\mathrm{L}}=\epsilon\left(m_{1}+m_{2}\right) \phi /(1+\phi)^{2}$ and the total ejecta mass $m_{\mathrm{e}}$ given by Eq. (41).

The drift velocity is characterized by the dimensionless parameter $\tilde{\tau}_{\text {stop }}$, where the scaled stopping time $\tilde{\tau}_{\text {stop }}$ is given by $a \Omega_{\mathrm{K}}^{2} \tau / u$. The relative velocity $u$ between a body and gas is equal to $\left(e^{*}+i^{*}+\eta\right) a \Omega_{\mathrm{K}}$. For $\tilde{\tau}_{\text {stop }} \lesssim 1$ and $\tilde{\tau}_{\text {stop }} \gg 1$, the drift velocity can be written as (Adachi et al. 1976, Inaba et al. 2001)

$$
v_{\text {drift }}(m, a)= \begin{cases}-\frac{2 \eta a \Omega_{\mathrm{K}}}{\tilde{\tau}_{\text {stop }}} \frac{\tilde{\tau}_{\text {stop }}^{2}}{1+\tilde{\tau}_{\text {stop }}^{2}} & \text { for } \quad \tilde{\tau}_{\text {stop }} \lesssim 1, \\ -2 \frac{a \eta}{\tau}\left\{\frac{[2 E(3 / 4)+K(3 / 4)]^{2}}{9 \pi^{2}} e^{* 2}+\frac{4}{\pi^{2}} i^{* 2}+\eta^{2}\right\}^{1 / 2} & \text { for } \quad \tilde{\tau}_{\text {stop }} \gg 1,\end{cases}
$$

where $E$ and $K$ are the elliptic integrals. Both regimes in Eq. (32) can be combined into

$$
v_{\text {drift }}(m, a)=-2 \frac{a \eta}{\tau} \frac{\tilde{\tau}_{\text {stop }}^{2}}{1+\tilde{\tau}_{\text {stop }}^{2}}\left\{\frac{[2 E(3 / 4)+K(3 / 4)]^{2}}{9 \pi^{2}} e^{* 2}+\frac{4}{\pi^{2}} i^{* 2}+\eta^{2}\right\}^{1 / 2} .
$$

Indeed, if $\tilde{\tau}_{\text {stop }} \lesssim 1$, then $e^{*}$ and $i^{*}$ are damped by the strong gas drag to $e^{*} \ll \eta$ and $i^{*} \ll \eta$, and Eq. (33) reduces to the first of Eqs. (32). If $\tilde{\tau}_{\text {stop }} \gg 1$, Eq. (33) simply coincides with the second of Eqs. (32).

Since we treat small fragments, we consider three gas-drag regimes: quadratic, Stokes, and Epstein ones. The gas drag coefficients $C_{\mathrm{D}}$ for these regimes are given by (Adachi et al. 1976)

$$
C_{\mathrm{D}, \mathrm{quad}}=0.5, \quad C_{\mathrm{D}, \text { Stokes }}=24 / R e, \quad C_{\mathrm{D}, \text { Epstein }}=16 / \mathrm{KRe},
$$

where $K=1.7\left(10^{-9} \mathrm{~g} \mathrm{~cm}^{-2} / \rho_{\text {gas }} r\right)$ is the Knudsen number and $R e=4.4(u / c) K^{-1}$ is the Reynolds number with $c$ being the sound velocity of gas (Adachi et al. 1976). All three 
regimes can be described together by adopting

$$
C_{\mathrm{D}}=C_{\mathrm{D}, \text { quad }}+\left(\frac{1}{C_{\mathrm{D}, \text { Stokes }}}+\frac{1}{C_{\mathrm{D}, \text { Epstein }}}\right)^{-1} .
$$

Since the mean collision rate is a function of $e^{*}$ and $i^{*}$, the evolution of $e^{*}$ and $i^{*}$ should be calculated precisely. We consider gravitational perturbations from other bodies, collisional damping, and gas drag. Their net effect can be calculated as a square of dispersions:

$$
\begin{aligned}
& \frac{d e^{* 2}}{d t}=\left(\frac{d e^{* 2}}{d t}\right)_{\text {grav }}+\left(\frac{d e^{* 2}}{d t}\right)_{\text {gas }}+\left(\frac{d e^{* 2}}{d t}\right)_{\text {coll }}, \\
& \frac{d i^{* 2}}{d t}=\left(\frac{d i^{* 2}}{d t}\right)_{\text {grav }}+\left(\frac{d i^{* 2}}{d t}\right)_{\text {gas }}+\left(\frac{d i^{* 2}}{d t}\right)_{\text {coll }},
\end{aligned}
$$

where terms with subscripts "grav", "gas", and "coll" indicate the time variation due to gravitational perturbations, gas drag, and collisions, respectively. These terms are provided by Inaba et al. (2001). Note that for the collisional damping, both fragments and mergers resulting from a collision have the velocity dispersion at the gravity center of colliding bodies, as described in their Eq. (30). We follow the planetary growth by simultaneously integrating Eqs. (30), (36), and (37).

Kokubo and Ida (1998) and Weidenschilling et al. (1997) showed that the radial separation of orbits of runaway bodies formed in a swarm of planetesimals is about 10 times their mutual Hill radius. Since dynamical friction from the field planetesimals makes orbits of the runaway bodies nearly circular and coplanar, the orbital crossing of these runaway bodies never occurs before the gas is dispersed (Iwasaki et al. 2001). Therefore, they cannot collide with each other. In our simulation, when the bodies reach a certain mass $m_{\text {run }}$ such that the sum of their mutual Hill radii equals the radial-bin width divided by $\tilde{b}$, the bodies are regarded as "runaway bodies" which do not have any collisions and dynamical interaction due to close encounters among them, following Inaba et al. (2001). We set $\tilde{b}=10$. As the bodies grow, their separation measured in the Hill radii decreases 
and these bodies can no longer be treated as "runaway bodies". Therefore, $m_{\text {run }}$ increases during the simulation and then the number of the "runaway bodies" decreases.

Moreover, Inaba et al. (2001) set the number of collisions during a numerical time interval to be an integer using a random number generator and hence keep the number of bodies an integer. Our procedure is different. Instead of dealing with the number of bodies, we treat the mathematical expectation $N$ of the number of bodies with mass larger than $m$. When $N$ is much lower than unity, the bodies may not yet exist. We introduce a certain critical number $N_{\mathrm{c}}$ (a "threshold") to get rid of such bodies. If $N\left(m_{\mathrm{c}}\right)=N_{\mathrm{c}}$, we neglect collisions with bodies larger than $m_{\mathrm{c}}$ and dynamical interactions with them. The value of $N_{\mathrm{c}}$ should be of the order of unity, because we can say that bodies are not yet born for $N \ll 1$, whereas choosing a high $N_{\text {c }}$ would delay the embryo growth. Bodies with masses ranging from $m_{\text {run }}$ to $m_{\mathrm{c}}$ are treated as "runaway bodies". The mass range of the "runaway bodies" tends to extend during the simulation for low $N_{\mathrm{c}}$, although being kept small in $N$-body simulation (Kokubo and Ida 2002). We always start the simulation with $N_{\mathrm{c}}=0.1$ and $N_{\mathrm{c}}$ may change from 0.1 to 10 during the simulation to keep the small mass range of the "runaway bodies". As shown in Figs. 1 and 2, our simulation with such a choice of $N_{\text {c }}$ reproduces the mass and velocity-dispersion distributions obtained from the $N$-body simulation, presented in Figs. 4 and 5 of Inaba et al. (2001). Where about 100 runs of the statistical code by Inaba et al. (2001) are required to reproduce the $N$-body simulation, our simulation does the same with only one run. 


\subsection{EMBRYO GROWTH}

\subsubsection{WITHOUT FRAGMENTATION}

We calculate the evolution of the number and velocity dispersion of bodies by summing up the time variations coming from all mass and radial bins and by integrating them over time. We integrate Eq. (30) through the fourth order of Runge-Kutta method for the mass evolution and Eqs. (36) and (37) through the linear method for $e^{*}$ and $i^{*}$ evolution. We set the mass radio between adjacent mass bins to 1.2, which we found sufficient to reproduce $N$-body simulations (see Figs. 1 and 2). Fig. 3 shows the results ignoring fragmentation $\left(Q_{\mathrm{D}}^{*}=\infty\right)$ with a set of six concentric annuli at $3.2,4.5,6.4,9.0,13$, and $18 \mathrm{AU}$ for $M_{*}=M_{\odot}, \Sigma_{1}=7 \mathrm{~g} / \mathrm{cm}^{2}$, and $q=3 / 2$. We consider planetesimals with initial mass of $m_{0}=4.2 \times 10^{18} \mathrm{~g}$ (radius of $r_{0}=10 \mathrm{~km}$ for $\rho=1 \mathrm{~g} / \mathrm{cm}^{3}$ ) and velocity dispersion given by $e^{*}=2 i^{*}=\left(2 m_{0} / M_{*}\right)^{1 / 3}$. The planetesimals are assumed to be composed of ice whose physical parameters are listed in Table 1 . We artificially apply the gas surface density evolution in the form $\Sigma_{\text {gas }}=\Sigma_{\text {gas }, 0} \exp \left(-t / T_{\text {gas,dep }}\right)$, where $T_{\text {gas,dep }}$ is the gas depletion timescale1 1 . Here, we set $T_{\text {gas,dep }}=10^{7}$ years.

Figure 3 a shows the mass distribution of bodies at $3.2 \mathrm{AU}$ from $10^{4}$ years to $4 \times 10^{6}$ years. After $10^{5}$ years, the mass distribution of small bodies $\left(m \lesssim 10^{24} \mathrm{~g}\right)$ is a single power law, which is consistent with that of the runaway growth, $n_{\mathrm{c}} \propto m^{-5 / 3}$ (Makino et al. 1998). At the same time, the oligarchic growth results in the flatter distribution for large bodies ( $\left.m \gtrsim 10^{24} \mathrm{~g}\right)$. Such large bodies have almost a single mass $\left(\sim 3 \times 10^{26} \mathrm{~g}\right.$ at $10^{6}$ years and $\sim 2 \times 10^{27} \mathrm{~g}$ at $4 \times 10^{6}$ years). As the growth of the large bodies proceeds, the random velocities of small bodies exceed the escape velocity because of the viscous stirring by large

\footnotetext{
${ }^{1}$ Assuming a constant $\Sigma_{\text {gas }}$ gives almost the same results for the final embryo mass, because we consider time spans $t \leq T_{\text {gas,dep }}$.
} 
bodies in the entire mass range of small bodies $\left(m \lesssim 10^{24} \mathrm{~g}\right)$. Then, the mass distribution starts to depart from this power law. Collisions between small bodies at such velocities lead to a flat mass distribution for small bodies $\left(m \lesssim 10^{21} \mathrm{~g}\right.$ at $10^{6}$ years and $m \lesssim 10^{22} \mathrm{~g}$ at $4 \times 10^{6}$ years) and a steep one for large bodies. This means that planetesimals dominating the surface density become larger after $10^{5}$ years.

The velocity dispersion evolution $\left(e^{*}\right.$ and $\left.i^{*}\right)$ at $3.2 \mathrm{AU}$ is shown in Fig. 4. At $10^{4}$ years, $e^{*}$ and $i^{*}$ are proportional to $m^{-1 / 2}$, being determined by the dynamical friction. At $10^{5}$ years, $e^{*}$ and $i^{*}$ for $m \lesssim 10^{22} \mathrm{~g}$ are independent of mass, whereas $e^{*}$ and $i^{*}$ of larger bodies still preserve an $m^{-1 / 2}$ dependence. The viscous stirring by large bodies dominates $e^{*}$ and $i^{*}$ of small bodies. In this case, $e^{*}$ is given by Eq. A1). After $10^{6}$ years, the velocity

dispersion of small bodies $\left(m \lesssim 10^{22} \mathrm{~g}\right.$ at $10^{6}$ years and $m \lesssim 10^{25} \mathrm{~g}$ at $4 \times 10^{6}$ years) reaches the equilibrium between the stirring and the gas drag. In this case, $e^{*}$ is given by Eq. (15). However, bodies with intermediate mass do not yet have the velocity dispersion in the equilibrium. The velocity dispersion of bodies dominating the surface density is still given by Eq. (A1). The growth of large bodies are described by $M_{\mathrm{n}}$ given by Eq. ( years (see Appendix A).

In the outer region, the mass distribution and velocity dispersion evolve in a similar way but in a longer timescale. At $4 \times 10^{6}$ years, the bodies with $m \gtrsim 10^{24} \mathrm{~g}$ grow through the oligarchic mode at $a \lesssim 9$ AU. Beyond 9 AU, the largest bodies have not yet reached $10^{24} \mathrm{~g}$ by that time.

\subsubsection{WITH FRAGMENTATION}

We now take into account fragmentation, using $Q_{\mathrm{D}}^{*}$ in Eq. (5). Kenyon and Bromley (2009) suggested that the small bodies coupled with gas $(\lesssim 1 \mathrm{~m})$ affect the embryo growth. 
To treat the coupled bodies, we set the smallest mass to be $4.2 \mathrm{~g}$ (radius of $1 \mathrm{~cm}$ for $\left.\rho=1 \mathrm{~g} / \mathrm{cm}^{3}\right)$.

The mass distribution and velocity evolution that we calculated with fragmentation are shown in Figs. 5 and 6. During the first $10^{5}$ years, bodies grow through mutual collisions. Fragments $\left(m \lesssim m_{0}\right)$ are not yet numerous because of the low velocity dispersion (Fig. 6). At $t=10^{6}$ years, the runaway growth occurs inside 6.4 AU. Similar to the case without fragmentation, the cumulative number $n_{\mathrm{c}}$ is almost proportional to $m^{-5 / 3}$ for $m \gtrsim 10^{20} \sim 100 m_{0}$ and the distribution of large bodies with $m \gtrsim 10^{24} \mathrm{~g}$ is flat. Since the velocity dispersion of planetesimals $\sim e^{*} v_{\mathrm{K}}$ exceeds their escape velocity for $m \lesssim 10^{24} \mathrm{~g}$, planetesimals dominating the surface density become larger after $10^{6}$ years. In contrast to the case without fragmentation, the mass of bodies dominating the surface density ceases at about $100 m_{0}$, owing to a high collisional velocity $e^{*} v_{\mathrm{K}} \gtrsim \sqrt{Q_{\mathrm{D}}^{*}}$. The slope of $n_{\mathrm{c}}$ for $m \sim 10^{11}-10^{20} \mathrm{~g}(r=0.1-10 \mathrm{~km})$ is nearly $-(5+3 p) /(6+3 p)$, which is typical of a collision cascade for $v^{2} / Q_{\mathrm{D}}^{*} \propto m^{-p}$ (Kobayashi and Tanaka 2010). The downward mass flux along the mass coordinate is constant with mass in the collision cascade. For $m \lesssim 10^{11} \mathrm{~g}$, the velocity dispersions $\left(e^{*}\right.$ and $\left.i^{*}\right)$ are effectively damped by the gas drag in the Stokes regime (See Fig. 6). Because the collisional energy $\left(\sim e^{* 2} v_{\mathrm{K}}^{2}\right)$ is lower than the energy threshold $\left(Q_{\mathrm{D}}^{*}\right)$ for a destructive collision, the downward mass flux becomes insignificant compared to that in the collision cascade. Therefore, the number of the bodies increases around $10^{11} \mathrm{~g}$ where $e^{* 2} v_{\mathrm{K}}^{2} \sim Q_{\mathrm{D}}^{*}$. Bodies with $m \sim 10^{5} \mathrm{~g}(r \sim 1 \mathrm{~m})$ are most efficiently removed by the radial drift and smaller bodies $\left(m \ll 10^{5}\right.$ g or $\left.r \ll 1 \mathrm{~m}\right)$ are coupled with gas. As a result, the mass distribution becomes wavy (see Fig. [5). The surface density of bodies with $e^{* 2} v_{\mathrm{K}}^{2} \sim Q_{\mathrm{D}}^{*}$ is much higher than that of the coupled bodies. However, planetesimals with $\sim 100 m_{0}$ dominate the surface density. At $4 \times 10^{6}$ years, the oligarchic growth is inhibited by fragmentation inside $5 \mathrm{AU}$, where the number of fragments decreases compared to that at $10^{6}$ years. 
Figure 7 shows the embryo-mas: 2 evolution at 3.2 AU. At the runaway growth phase (the first $\lesssim 10^{5}$ years), the embryo mass grows as an exponential function of time. The growth rate becomes much lower in the oligarchic mode at $10^{5}-10^{6}$ years. The embryos exceed that for the case without fragmentation. They are substantially fed by small fragments whose velocities are damped by gas drag and are low (see Figs. 6), resulting in their rapid growth. However, they grow at a sluggish pace at about $4 \times 10^{5}$ years because the surface density of planetesimals is decreased by collision cascade followed by removal of small bodies by gas drag. As a result, the final embryo mass is reduced by fragmentation. In this case, since $M_{\mathrm{c}}$ and $M_{\mathrm{f}}$ are comparable, the embryo mass is determined by both effects. Even so, the final embryo mass is consistent with $M_{\mathrm{c}}$ and $M_{\mathrm{f}}$.

Fig. 8 presents the embryo mass at $10^{7}$ years as a function of distance, including the results for three additional annuli at $1,1.4$, and $2.0 \mathrm{AU} 3$ In simulations at $a \leq 2.7 \mathrm{AU}$, we use silicate bodies (Table 1) instead of icy bodies. Decline of the planetesimal surface density caused by fragmentation results in embryos smaller than those for the case without fragmentation inside $6 \mathrm{AU}$. The masses reach only $0.01-0.1 M_{\oplus}$ in $10^{7}$ years. The embryo masses at $a \lesssim 1.5 \mathrm{AU}$ and $a=2.7-5 \mathrm{AU}$ are consistent with $M_{\mathrm{c}}$ or $M_{\mathrm{f}}$. Inside $a \lesssim 1.5 \mathrm{AU}$, the final mass is determined by the largest of the two masses, $M_{\mathrm{c}}$ and $M_{\mathrm{f}}$ (see Fig. 9). For $m_{0}=4.2 \times 10^{15} \mathrm{~g}\left(r_{0}=1 \mathrm{~km}\right)$, the embryo mass is determined by $M_{\mathrm{f}}$ because of $M_{\mathrm{f}}>M_{\mathrm{c}}$ (see Fig. 10). In addition, if both $M_{\mathrm{c}}$ and $M_{\mathrm{f}}$ are smaller than $M_{\mathrm{n}}$ in Eq. (A3), the fragmentation is nearly negligible and the embryo mass is given by $M_{\mathrm{n}}$ (see Appendix A for an analysis of embryo growth without fragmentation).

Since the initial mass of planetesimals depends on their formation process, which is not well understood, it is worthwhile investigating the embryo mass dependence on the initial

\footnotetext{
${ }^{2}$ Here, embryo mass represents the average mass of the "runaway bodies".

${ }^{3}$ The results come from two simulations: one in $a<2.7 \mathrm{AU}$ and the other in $a>2.7 \mathrm{AU}$.
} 
planetesimal mass. Fig. 11 shows the dependence of the embryo masses on $m_{0}$ at $3.2 \mathrm{AU}$ for the minimum mass solar nebula after 10 million years. If the initial mass is smaller than $\sim 10^{17} \mathrm{~g}$, the embryo mass is determined by accretion of small fragments. For larger $m_{0}$, growth is halted by the collision cascade. The embryo mass increases with $m_{0}$ because the timescale of the surface density decline due to collision cascade is longer for larger planetesimals, strengthened by self-gravity (large $Q_{\mathrm{D}}^{*}$ ). If $m_{0} \gtrsim 10^{21} \mathrm{~g}$, the embryo grows without substantial fragmentation. For a massive disk $\left(\Sigma_{1}=70 \mathrm{~g} / \mathrm{cm}^{2}\right)$, the embryo mass can reach the Earth mass. In this case, $M_{\mathrm{c}}$ and $M_{\mathrm{f}}$ determine the embryo mass because of the short growth time for $r_{0}=1-100 \mathrm{~km}$.

\section{SUMMARY AND DISCUSSION}

In this paper, we studied analytically and numerically the growth of planetary embryos in the framework of the standard scenario. We took into account that embryos growing in the oligarchic mode pump up relative velocities of planetesimals, which causes their collisional fragmentation. We also considered the fate of smallest fragments of the resulting collision cascade, namely their inward drag in the ambient gas and possible accretion by nascent embryos.

Taking into account fragmentation, we have analytically derived the final mass of a planetary embryo in Section 2 in two cases: the mass $M_{\mathrm{c}}$ of an embryo growing through the accretion of planetesimals which are removed by collision cascade and the mass $M_{\mathrm{f}}$ in the case where an embryo grows through collisions with fragments which are removed by gas drag. In Sec. 3, we showed that the final embryo mass obtained in numerical simulations can be reproduced by the larger of the two analytic estimates, $\operatorname{MAX}\left(M_{\mathrm{c}}, M_{\mathrm{f}}\right)$. If the embryo mass $M_{\mathrm{n}}$ of Eq. (A3) for the case without fragmentation is smaller than $\operatorname{MAX}\left(M_{\mathrm{c}}, M_{\mathrm{f}}\right)$, fragmentation is negligible and the final mass is given by $M_{\mathrm{n}}$. Altogether, our analytical 
formulae provide a good estimate of the final mass of planetary embryos.

The gas giant planet formation via core accretion requires the solid core as large as $\sim 10 M_{\oplus}$ (e.g., Ikoma et al. 2001). Kenyon and Bromley (2009) suggested that the planetary embryo may further grow by the accretion of bodies coupled with gas because the coupled bodies are no longer involved in collision cascade. Taking into account three regimes of gas drag (see Eq. 35), we found that the collision cascade halts at bodies having $v^{2} \sim Q_{\mathrm{D}}^{*}$, i.e. at sizes that are larger than the critical coupling size corresponding to $\tilde{\tau}_{\text {stop }}=1$. As a result, coupled bodies should be produced only in little amounts and can hardly contribute to planetary growth, despite their low velocity dispersion in the laminar disks considered here.

In this paper, we derive the final embryo masses, assuming a uniform distribution of solid bodies. However, the planetary embryos can open gaps in a solid disk, which would affect the accretion of fragments on the embryo and their removal by the gas drag. Tanaka and Ida (1999) derived a condition for the gap opening around a migrating embryo. Replacing the embryo's migration time by the drift time $\tau / 2 \eta^{2}$ for fragments, we can obtain a criterion for an embryo with mass $M$ to open a gap in the solid disk from their Eq. (3.6),

$$
\frac{\tau \Omega_{\mathrm{K}} h_{M}^{2}}{4 \pi \eta^{2}} \geq 0.81\left[\sqrt{1+0.45\left(\tau \Omega_{\mathrm{K}} / 2 \pi\right)^{2 / 3}}+1\right]^{2},
$$

where $\tau$ is given by Eq. (14) for fragments surrounding the embryos. Since Eq. (38) requires $\tau \Omega_{\mathrm{K}} / 2 \pi \ll 1$ for $M \gtrsim 0.1 M_{\oplus}$, the critical fragment radius $r_{\mathrm{g}}$ above which Eq. (38) is satisfied is given by

$$
r_{\mathrm{g}} \simeq 1.0 \times 10^{2}\left(\frac{M}{0.1 M_{\oplus}}\right)^{-1 / 3}\left(\frac{a}{5 \mathrm{AU}}\right)^{3 / 8} \mathrm{~m},
$$

where we adopt $q=3 / 2$ and $C_{\mathrm{D}}=C_{\mathrm{C}, \text { Stokes. }}$. Levison et al. (2010) performed $N$-body simulations involving fragments and found $r_{\mathrm{g}} \sim 30 \mathrm{~m}$ for $M \geq M_{\oplus}$, which is consistent with Eq. (39). On the other hand, as we have shown, the solid surface density is reduced 
by the elimination of fragments at the low-mass end of collision cascade due to gas drag. In addition, the embryo grows through the accretion of the fragments for small initial planetesimals. The radius $r_{\mathrm{e}}$ of the fragments is estimated by Eq. (25) as

$$
r_{\mathrm{e}} \simeq 5.0\left(\frac{M}{0.1 M_{\oplus}}\right)^{-1 / 2}\left(\frac{a}{5 \mathrm{AU}}\right)^{1 / 2} \mathrm{~m} .
$$

Eqs. (39) and (40) give $r_{\mathrm{e}} \ll r_{\mathrm{g}}$. This means that the collision cascade quickly grinds planetesimals down to the size much less than $r_{\mathrm{g}}$. As a result, the gap formation in the solid disk composed of fragments with the radius $r_{\mathrm{e}}$ does not occur and the embryo growth would not be influenced.

Considering the range of initial planetesimal radii $r_{0}=1-100 \mathrm{~km}$, and the range of disk surface densities $\Sigma_{1} \sim 7-70 \mathrm{~g} / \mathrm{cm}^{2}$ at $3.2 \mathrm{AU}(1-10 \times \mathrm{MMSN})$, our results suggest that the fragmentation averts the planetary growth at $M \sim 0.1-10 M_{\oplus}$ at several AU. This is consistent with the results of planetary growth simulations beyond $30 \mathrm{AU}$ by Kenyon and Bromley (2008). The cores approaching the critical mass of $10 M_{\oplus}$ can only form at $a=3-4 \mathrm{AU}$, only from planetesimals that have $r_{0} \sim 100 \mathrm{~km}$ initially, and only in densest nebula with $\Sigma_{1} \sim 70 \mathrm{~g} / \mathrm{cm}^{2}$. Thus it appears problematic to explain formation of giant planets at 5-10 AU, such as Jupiter and Saturn in the solar system, even for $\Sigma_{1} \sim 70 \mathrm{~g} / \mathrm{cm}^{2}$ and $r_{0} \sim 100 \mathrm{~km}$.

An effect that could help further is the enhancement of the embryo's collisional cross section due to a tenuous atmosphere acquired by the embryo in the ambient gas. Inaba and Ikoma (2003) and Inaba et al. (2003) have shown that a core with mass exceeding about $0.1 M_{\oplus}$ could grow by that effect to the critical core mass. As found here, the embryo masses can indeed be about $0.1 M_{\oplus}$ under the minimum mass solar nebula conditions, at least for an initial planetesimal radius greater than $10 \mathrm{~km}$.

On any account, our results strongly suggest that increasing the disk surface density and/or the initial embryo size helps forming larger cores. This brings up the question 
whether the values that we need to grow the embryo to the critical mass, $10 \times \mathrm{MMSN}$ and $r_{0} \sim 100 \mathrm{~km}$ - or less, if the atmospheric effect is taken into account — appear plausible. As far as the disk density is concerned, the answer is probably yes. $10 \times \mathrm{MMSN}$ is close to the gravitational stability limit, and all densities below this limit cannot be ruled out. For instance, Desch (2007) and Crida (2009) point out that the MMSN profile is inconsistent with the "Nice model" (Gomes et al. 2004) and should be replaced with another surface density profile, which would possibly imply larger surface densities at several AU.

We now consider the initial size of planetesimals. The mechanisms of planetesimal formation are highly debated but, despite intensive effort, remain fairly unknown. Classical models in which dust smoothly grows to planetesimals with $r_{0} \sim 1 \mathrm{~km}$ face the "meter barrier" problems: first, meter-sized objects should be lost to the central star as a result of gas drag (Weidenschilling 1977, Brauer et al. 2008), and second, further agglomeration of meter-sized objects upon collision is problematic (Blum and Wurm 2008). Accordingly, in the last years, competing scenarios were suggested that circumvent these barriers, such that the "primary accretion" mechanism proposed by Cuzzi et al. (2008) and "graviturbulent" formation triggered by transient zones of high pressure (Johansen et al. 2006) or by streaming instabilities (Johansen et al. 2007). These models all imply rapid formation of rather large planetesimals in the $r_{0} \sim 100-1000 \mathrm{~km}$ range. Support for these scenarios may come from the analysis of left-over planetesimals in the solar system. Morbidelli et al. (2009), for instance, suggest that the initial planetesimals should be larger than $100 \mathrm{~km}$ to reproduce the mass distribution of asteroids in the main belt.

Our last remark is related to the Type I migration of bodies due to interaction with gas (e.g., Tanaka et al. 2002). The planetary embryo grows in the runaway mode followed by the oligarchic one. The timescale of the runaway growth is proportional to the initial size of bodies. In addition, the embryos rapidly grow through collisions with small fragments for 
small $r_{0}$ even in the the oligarchic growth. The embryo eventually grows to a larger mass if it is large initially, while it forms earlier if it starts with a smaller mass. Therefore, initially small planetesimals seem to be preferred for the core formation prior to their removal due to Type I migration. However, this is only valid for laminar disks. Turbulence may help prevent the loss of growing embryos to Type I migration (e.g., Laughlin et al. 2004). So, the alternative scanarios of planetesimal formation that all require turbulent disks may help here, too.

\section{ACKNOWLEDGMENT}

We thank Glen R. Stewart and an anonymous referee for their thorough reviews of this paper. H. K. and A. V. K. are grateful to Martin Ilgner for helpful comments. 


\section{A. PLANETARY GROWTH WITHOUT FRAGMENTATION}

When the growth timescale $T_{\text {grow }}$ of embryos is much longer than the $e^{*}$-damping timescale $T_{\text {gas }}$ by gas drag, $e^{*}$ settles to the equilibrium between the stirring and the gas drag and is given by Eq. (15). However, the growth timescale at the beginning of oligarchic growth is relatively short. In this case, $e^{*}$ cannot reach the equilibrium for $T_{\text {grow }} \ll T_{\text {gas }}$. Using these two limits of $e^{*}$, we will derive the embryo mass evolution as follows.

In the non-equilibrium case $\left(T_{\text {grow }} \ll T_{\text {gas }}\right)$, $e^{*}$ increases as $M$ grows. Then, combining the growth rate (Eqs. (8) -(9)) with the stirring rate (Eq. (10) with Eqs. (11) and (12)), we get

$$
e^{*}=\left[\frac{3 C_{\mathrm{VS}} \ln \left(\Lambda^{2}+1\right) M^{4 / 3}}{2^{10 / 3} \pi C_{\mathrm{acc}} \tilde{b} a^{2} \Sigma_{\mathrm{s}} \tilde{R} C_{\mathrm{col}}\left(3 M_{*}\right)^{1 / 3}}\right]^{1 / 2},
$$

where we assume that the initial embryo mass and eccentricity dispersion are much smaller than $M$ and $e^{*}$, respectively. Inserting Eq. (A1) into Eq. (8) with Eq. (91) there results

$$
\frac{d M}{d t}=\frac{2^{10 / 3} \pi \tilde{b}\left(C_{\mathrm{acc}} \Sigma_{\mathrm{s}} C_{\mathrm{col}} a^{2} \tilde{R}\right)^{2} \Omega_{\mathrm{K}}}{9 M_{*} C_{\mathrm{VS}} \ln \left(\Lambda^{2}+1\right)} .
$$

If the embryo grows with this rate for sufficiently long time, it is valid use $M=0$ at $t=0$ as the initial condition. In this case, the embryo mass $M_{\mathrm{n}}$ at time $t$ is given by

$$
M_{\mathrm{n}}=\frac{2^{10 / 3} \pi \tilde{b}\left(C_{\mathrm{acc}} \Sigma_{\mathrm{s}} C_{\mathrm{col}} a^{2} \tilde{R}\right)^{2} \Omega_{\mathrm{K}} t}{9 M_{*} C_{\mathrm{VS}} \ln \left(\Lambda^{2}+1\right)},
$$

which is independent of $m$.

In the equilibrium case $\left(T_{\text {grow }} \gg T_{\text {gas }}\right)$, the growth rate of $M$ is given by Eq. (16). Also we consider that the embryo mass becomes much larger than the initial mass. Integrating Eq. (16) over time with $M=0$ at $t=0$, we have

$$
M_{\mathrm{e}}=\left[\frac{C_{\mathrm{acc}} \Sigma a^{2} C_{\mathrm{col}} \tilde{R} \Omega_{\mathrm{K}}}{3\left(3 M_{*}\right)^{2 / 3}}\right]^{3}\left[\frac{2^{4 / 3} \pi \tilde{b} C_{\mathrm{gas}}}{C_{\mathrm{VS}} \ln \left(\Lambda^{2}+1\right) \Omega_{\mathrm{K}} \tau}\right]^{6 / 5} t^{3} .
$$


The embryo mass is proportional to $m^{2 / 5}$ from the $m$ dependence of $\tau$. The growth time of embryo mass $M_{\mathrm{e}}$ is similar to that estimated by Kokubo and Ida (2002).

Fig. 13 shows the embryo growth at 3.2 AU. The runaway growth swithes to oligarchic growth after $10^{5}$ years. The embryo mass is consistent with $M_{\mathrm{n}}$ given by Eq. (A3) in $10^{5}-10^{7}$ years. On contrary, the embryo mass does not agree with $M_{\mathrm{e}}$ given by Eq. (A4). That is because $e^{*}$ of the representative planetesimal, which dominates the surface density, does not reach the equilibrium. As seen in Fig. 3, the representative planetesimal grows because its random velocity is higher than its surface escape velocity. Although $e^{*}$ reaches the equilibrium for small bodies, that of the representative planetesimal cannot do due to the growth. Since the representative planetesimal growth is caused by the perfect sticking even for a high velocity, the growth came from the artificial treatment. In practice, the growth halts at $\sim 100 m_{0}$ when fragmentation is included, because the impact energy exceeds $Q_{\mathrm{D}}^{*}$. In this case, $e^{*}$ of the representative planetesimals reach the equilibrium and the embryo mass is determined by $M_{\mathrm{c}}$ or $M_{\mathrm{f}}$ because of the active fragmentation.

\section{B. MASS DEPLETION DUE TO COLLISION CASCADE}

Here, we derive the surface-density depletion rate due to collision cascade, following Kobayashi and Tanaka (2010). Focusing on the mass transport by fragmentation, we neglect terms in the right-hand side of Eq. (30) except for the third one:

$$
\begin{aligned}
\frac{\partial m n_{\mathrm{s}}}{\partial t}=\frac{\partial}{\partial m} \Omega_{\mathrm{K}} \int_{m}^{\infty} d m_{1} \int_{0}^{m_{1}} d m_{2}\left(m_{1}+m_{2}\right) f\left(m, m_{1}, m_{2}\right) \\
\quad \times n_{\mathrm{s}}\left(m_{1}, a\right) n_{\mathrm{s}}\left(m_{2}, a\right)\left(h_{m_{1}, m_{2}} a\right)^{2}\left\langle P_{\mathrm{col}}\right\rangle_{m_{1}, m_{2}}
\end{aligned}
$$

Collisional velocities exceed the surface escape velocity of colliding bodies in collision

cascade, resulting in $\left(h_{m_{1}, m_{2}} a\right)^{2}\left\langle P_{\text {col }}\right\rangle=(17.3 / 2 \pi)\left(r_{1}+r_{2}\right)^{2}=h_{0} m_{1}^{2 / 3}\left[1+\left(m_{2} / m_{1}\right)^{1 / 3}\right]^{2}$. Here, $r_{1}$ and $r_{2}$ are the radii of $m_{1}$ and $m_{2}$, respectively. Since collisions with $m_{1} \gg m_{2}$ 
mainly lead to the mass transport along the mass coordinate in collision cascade, the $f$ function given by Eq. (31) becomes

$$
f\left(m, m_{1}, m_{2}\right)= \begin{cases}\left(\frac{m}{m_{1}}\right)^{2-b} \frac{\phi}{1+\phi}\left[\frac{\epsilon \phi}{(1+\phi)^{2}}\right]^{b-2} & \text { for } \frac{m}{m_{1}}<\frac{\epsilon \phi}{(1+\phi)^{2}} \\ \frac{\phi}{1+\phi} & \text { for } \frac{m}{m_{1}} \geq \frac{\epsilon \phi}{(1+\phi)^{2}}\end{cases}
$$

Furthermore, $\left(h_{m_{1}, m_{2}} a\right)^{2}\left\langle P_{\text {col }}\right\rangle=h_{0} m_{1}^{2 / 3}$ and $\phi=\left(v\left(m_{1}\right)^{2} / 2 Q_{\mathrm{D}}^{*}\left(m_{1}\right)\right)\left(m_{2} / m_{1}\right)$. Introducing the dimensionless variable $x=m / m_{1}$, we can re-write Eq. (B1) as

$$
\begin{aligned}
\frac{\partial m n_{\mathrm{s}}}{\partial t}=\frac{\partial}{\partial m} & A^{2} \Omega_{\mathrm{K}} h_{0} m^{\frac{11}{3}-2 \alpha}\left(\frac{v^{2}(m)}{2 Q_{\mathrm{D}}^{*}(m)}\right)^{\alpha-1} \int_{0}^{v^{2}\left(m_{1}\right) / 2 Q_{\mathrm{D}}^{*}\left(m_{1}\right)} d \phi \frac{\phi^{1-\alpha}}{1+\phi} \\
\times & {\left[\int_{0}^{\epsilon \phi /(1+\phi)^{2}} d x x^{2 \alpha-8 / 3+(\alpha-1) p-b}\left(\frac{\epsilon \phi}{(1+\phi)^{2}}\right)^{b-2}\right.} \\
& \left.+\int_{\epsilon \phi /(1+\phi)^{2}}^{1} d x x^{2 \alpha-14 / 3+(\alpha-1) p}\right],
\end{aligned}
$$

where $n_{\mathrm{s}}=A m^{-\alpha}$ and $v^{2}(m) / Q_{\mathrm{D}}^{*}(m) \propto m^{-p}$. The upper integration limit over $\phi$ in Eq. (B3) is different from Eq. (31) of Kobayashi and Tanaka (2010), which comes from the different definition of $f$. Despite that, Eq. (17) is consistent with Kobayashi and Tanaka because of a insignificant contribution of collisions with $\phi>v^{2}\left(m_{1}\right) / 2 Q_{\mathrm{D}}^{*}\left(m_{1}\right)$ to the integral over $\phi$ in their Eq. (31). Since $v^{2}\left(m_{1}\right) / 2 Q_{\mathrm{D}}^{*}\left(m_{1}\right) \gg 1$, we change the upper limit to $\infty$. We also take into account that $v^{2}(m) / Q_{\mathrm{D}}^{*}(m) \propto m^{-p}$ and that $\partial m n_{\mathrm{s}} / \partial t=0$ in a steady-state collision cascade. Then, Eq. (B33) gives $\alpha=(11+3 p) /(6+3 p)$. Note that Eqs.(31), (34), (B2), and (B3) of Kobayashi and Tanaka (2010) contain mistakes: their power-law exponents of $m$ should be $11 / 3-2 \alpha$, as in Eq. (B33).

In the oligarchic growth, since planetary embryos sufficiently increase the collisional velocity of the swarm planetesimals, collision cascade depletes the swarm. Smaller bodies quickly reach the steady state of collision cascade. Therefore, integrating Eq. (B3) over mass in the steady-state collision cascade $(\alpha=(11+3 p) /(6+3 p))$, we obtain

$$
\frac{\partial \Sigma_{\mathrm{s}}}{\partial t}=-A^{2} m_{\max }^{11 / 3-2 \alpha} \Omega_{\mathrm{K}} h_{0}\left(\frac{v^{2}\left(m_{\max }\right)}{2 Q_{\mathrm{D}}^{*}\left(m_{\max }\right)}\right)^{\alpha-1} \int_{0}^{\infty} d \phi \frac{\phi^{1-\alpha}}{1+\phi}
$$




$$
\times\left[\int_{0}^{\epsilon \phi /(1+\phi)^{2}} d x x^{1-b}\left(\frac{\epsilon \phi}{(1+\phi)^{2}}\right)^{b-2}+\int_{\epsilon \phi /(1+\phi)^{2}}^{1} d x x^{-1}\right]
$$

where $m_{\max }$ is the mass of largest planetesimals in collision cascade. Integrating over $x$

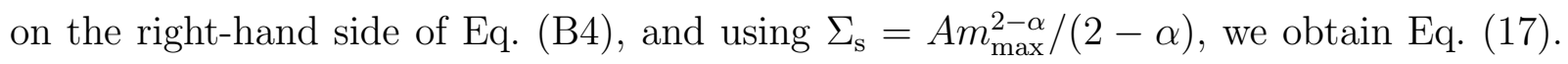
In Eq. (17), we convert $m_{\max }$ to $m$, according to the definition in Section 2, Moreover, Eq. (17) includes the additional terms due to the mass transport of the remnant according to Kobayashi and Tanaka, although the transport terms are much smaller than others.4

${ }^{4}$ The negative sign in the right-hand side of Eq. (A5) of Kobayashi and Tanaka (2010) is in error, and we correct this in Eq. (17). 


\section{REFERENCES}

Adachi, I., Hayashi, C., Nakazawa, K. 1976. The gas drag effect on the elliptical motion of a solid body in the primordial solar nebula.. Progress of Theoretical Physics 56, 1756-1771.

Benz, W., Asphaug, E. 1999. Catastrophic Disruptions Revisited. Icarus 142, 5-20.

Blum, J., Wurm, G. 2008. The Growth Mechanisms of Macroscopic Bodies in Protoplanetary Disks. Annual Review of Astronomy and Astrophysics 46, 21-56.

Bodenheimer, P., Pollack, J. B. 1986. Calculations of the accretion and evolution of giant planets The effects of solid cores. Icarus 67, 391-408.

Brauer, F., Dullemond, C. P., Henning, T. 2008. Coagulation, fragmentation and radial motion of solid particles in protoplanetary disks. Astronomy and Astrophysics 480, 859-877.

Bromley, B. C., Kenyon, S. J. 2006. A Hybrid N-Body-Coagulation Code for Planet Formation. Astronomical Journal 131, 2737-2748.

Chambers, J. 2008. Oligarchic growth with migration and fragmentation. Icarus 198, 256-273.

Crida, A. 2009. Minimum Mass Solar Nebulae and Planetary Migration. Astrophysical Journal 698, 606-614.

Cuzzi, J. N., Hogan, R. C., Shariff, K. 2008. Toward Planetesimals: Dense Chondrule Clumps in the Protoplanetary Nebula. Astrophysical Journal 687, 1432-1447.

Desch, S. J. 2007. Mass Distribution and Planet Formation in the Solar Nebula. Astrophysical Journal 671, 878-893. 
Gomes, R. S., Morbidelli, A., Levison, H. F. 2004. Planetary migration in a planetesimal disk: why did Neptune stop at 30 AU? Icarus 170, 492-507.

Hayashi, C. 1981. Structure of the Solar Nebula, Growth and Decay of Magnetic Fields and Effects of Magnetic and Turbulent Viscosities on the Nebula. Progress of Theoretical Physics Supplement 70, 35-53.

Hayashi, C., Nakazawa, K., Nakagawa, Y. 1985. Formation of the solar system. Protostars and Planets II 1100-1153.

Ikoma, M., Emori, H., Nakazawa, K. 2001. Formation of Giant Planets in Dense Nebulae: Critical Core Mass Revisited. Astrophysical Journal 553, 999-1005.

Inaba, S., Ikoma, M. 2003. Enhanced collisional growth of a protoplanet that has an atmosphere. Astronomy and Astrophysics 410, 711-723.

Inaba, S., Tanaka, H., Nakazawa, K., Wetherill, G. W., Kokubo, E. 2001. High-Accuracy Statistical Simulation of Planetary Accretion: II. Comparison with N-Body Simulation. Icarus 149, 235-250.

Inaba, S., Tanaka, H., Ohtsuki, K., Nakazawa, K. 1999. High-accuracy statistical simulation of planetary accretion: I. Test of the accuracy by comparison with the solution to the stochastic coagulation equation. Earth, Planets, and Space 51, 205-217.

Inaba, S., Wetherill, G. W., Ikoma, M. 2003. Formation of gas giant planets: core accretion models with fragmentation and planetary envelope. Icarus 166, 46-62.

Iwasaki, K., Tanaka, H., Nakazawa, K., Hiroyuki, E. 2001. The Gas-Drag Effect on the Orbital Instability of a Protoplanet System. Publications of the Astronomical Society of Japan 53, 321-329. 
Johansen, A., Klahr, H., Henning, T. 2006. Gravoturbulent Formation of Planetesimals. Astrophysical Journal 636, 1121-1134.

Johansen, A., Oishi, J. S., Low, M.-M. M., Klahr, H., Henning, T., Youdin, A. 2007. Rapid planetesimal formation in turbulent circumstellar disks. Nature 448, 1022-1025.

Kenyon, S. J., Bromley, B. C. 2004. Collisional Cascades in Planetesimal Disks. II. Embedded Planets. Astronomical Journal 127, 513-530.

Kenyon, S. J., Bromley, B. C. 2008. Variations on Debris Disks: Icy Planet Formation at 30-150 AU for 1-3 $M_{\odot}$ Main-Sequence Stars. Astrophysical Journal Supplement Series 179, 451-483.

Kenyon, S. J., Bromley, B. C. 2009. Rapid Formation of Icy Super-Earths and the Cores of Gas Giant Planets. Astrophysical Journal 690, L140-L143.

Kobayashi, H., Tanaka, H. 2010 Fragmentation Model Dependence of Collision Cascades. Icarus 206, 735-746.

Kokubo, E., Ida, S. 2002. Formation of Protoplanet Systems and Diversity of Planetary Systems. Astrophysical Journal 581, 666-680.

Kokubo, E., Ida, S. 2000. Formation of Protoplanets from Planetesimals in the Solar Nebula. Icarus 143, 15-27.

Kokubo, E., Ida, S. 1998. Oligarchic Growth of Protoplanets. Icarus 131, 171-178.

Kokubo, E., Ida, S. 1996. On Runaway Growth of Planetesimals. Icarus 123, 180-191.

Kusaka, T., Nakano, T., Hayashi, C. 1970. Growth of Solid Particles in the Primordial Solar Nebula. Progress of Theoretical Physics 44, 1580-1595. 
Laughlin, G., Steinacker, A., Adams, F. C. 2004. Type I Planetary Migration with MHD Turbulence. Astrophysical Journal 608, 489-496.

Levison, H. F., Thommes, E., Duncan, M. J. 2010. Modeling the Formation of Giant Planet Cores. I. Evaluating Key Processes. The Astronomical Journal 139, 1297-1314.

Makino, J., Fukushige, T., Funato, Y., Kokubo, E. 1998. On the mass distribution of planetesimals in the early runaway stage. New Astronomy 3, 411-416.

Mizuno, H., Nakazawa, K., Hayashi, C. 1980. Dissolution of the primordial rare gases into the molten earth's material. Earth and Planetary Science Letters 50, 202-210.

Morbidelli, A., Bottke, W. F., Nesvorný, D., Levison, H. F. 2009. Asteroids were born big. Icarus 204, 558-573.

Ohtsuki, K., Stewart, G. R., Ida, S. 2002. Evolution of Planetesimal Velocities Based on Three-Body Orbital Integrations and Growth of Protoplanets. Icarus 155, 436-453.

Pollack, J. B., Hubickyj, O., Bodenheimer, P., Lissauer, J. J., Podolak, M., Greenzweig, Y. 1996. Formation of the Giant Planets by Concurrent Accretion of Solids and Gas. Icarus 124, 62-85.

Stewart, S. T., Leinhardt, Z. M. 2009. Velocity-Dependent Catastrophic Disruption Criteria for Planetesimals. Astrophysical Journal 691, L133-L137.

Tanaka, H., Ida, S. 1999. Growth of a Migrating Protoplanet. Icarus 139, 350-366.

Tanaka, H., Takeuchi, T., Ward, W. R. 2002. Three-Dimensional Interaction between a Planet and an Isothermal Gaseous Disk. I. Corotation and Lindblad Torques and Planet Migration. Astrophysical Journal 565, 1257-1274.

Weidenschilling, S. J. 1977. Aerodynamics of solid bodies in the solar nebula. Monthly Notices of the Royal Astronomical Society 180, 57-70. 
Weidenschilling, S. J., Spaute, D., Davis, D. R., Marzari, F., Ohtsuki, K. 1997. Accretional Evolution of a Planetesimal Swarm. Icarus 128, 429-455.

Wetherill, G. W., Stewart, G. R. 1993. Formation of planetary embryos - Effects of fragmentation, low relative velocity, and independent variation of eccentricity and inclination. Icarus 106, 190.

Wetherill, G. W., Stewart, G. R. 1989. Accumulation of a swarm of small planetesimals. Icarus $77,330-357$.

This manuscript was prepared with the AAS LATEX macros v5.0. 
Table 1: Material properties

\begin{tabular}{c|cccccc} 
& $\begin{array}{c}Q_{0 \mathrm{~s}} \\
(\mathrm{erg} / \mathrm{g})\end{array}$ & $\beta_{\mathrm{s}}$ & $\begin{array}{c}Q_{0 \mathrm{~g}} \\
\left(\mathrm{erg} \mathrm{cm} / \mathrm{g}^{2}\right)\end{array}$ & $\beta_{\mathrm{g}}$ & $C_{\mathrm{gg}}$ & $\begin{array}{c}\rho \\
\left(\mathrm{g} / \mathrm{cm}^{3}\right)\end{array}$ \\
\hline ice & $7.0 \times 10^{7}$ & -0.45 & 2.1 & 1.19 & 9 & 1 \\
silicate & $3.5 \times 10^{7}$ & -0.38 & 0.3 & 1.36 & 9 & 3
\end{tabular}


Fig. 1 - Evolution of the mass distribution of bodies with $m_{0}=2 \times 10^{23} \mathrm{~g}, \rho=2 \mathrm{~g} / \mathrm{cm}^{2}$, and $e_{0}^{*}=2 i_{0}^{*}=0.002$ at $1 \mathrm{AU}$. The initial mass distribution is shown with the dotted curve in panel (a). Collisional fragmentation is neglected. Gray dashed lines represent the results of $N$-body simulation shown in Fig. 4 of Inaba et al. (2001).

Fig. 2] - Evolution of the velocity dispersion of bodies for the same conditions as in Fig. 1. The solid and dotted lines indicate $e^{*}$ and $i^{*}$, respectively. Circles and triangles represent $e^{*}$ and $i^{*}$ calculated by $N$-body simulation shown in Fig. 4 of Inaba et al. (2001), respectively.

Fig. 3 - The mass distribution of bodies at $10^{4}(\mathrm{~A}), 10^{5}(\mathrm{~B}), 10^{6}(\mathrm{C}), 4 \times 10^{6}(\mathrm{D})$ years, neglecting fragmentation. Different panels correspond to different radial annuli.

Fig. 4 - The velocity-dispersion distribution at $3.2 \mathrm{AU}$ after $10^{4}, 10^{5}, 10^{6}, 4 \times 10^{6}$ years of evolution. Fragmentation is neglected. The solid and dotted lines indicate $e^{*}$ and $i^{*}$, respectively.

Fig. 5 - Same as Fig. 3, but with fragmentation.

Fig. 6] - Same as Fig. 4, but with fragmentation.

Fig. 7 - Evolution of the embryo mass at 3.2 AU with fragmentation (black solid line) and without fragmentation (gray solid line). Dashed lines indicate $M_{\mathrm{c}}$ (Eq. 19; black) and $M_{\mathrm{f}}$ (Eq. 28; gray).

Fig. 8 - The embryo mass at $10^{7}$ years for initial planetesimal mass of $4.2 \times 10^{18} \mathrm{~g}$ with fragmentation (filled circles) and without fragmentation (open squares). Dashed lines indicate $M_{\mathrm{c}}$ (Eq. 19, black) and $M_{\mathrm{f}}$ (Eq. 20, grey). Gray solid line shows $M_{\text {iso }}$ (Eq. 6) for comparison. The vertical line represents the snow line $a=a_{\text {ice }}=2.7 \mathrm{AU}$.

Fig. 9 - Same as Fig. 7, but at 1 AU. 
Fig. 10 - Same as Fig. 7, but for $m_{0}=4.2 \times 10^{15} \mathrm{~g}\left(r_{0}=1 \mathrm{~km}\right)$.

Fig. 11 - The embryo mass at $3.2 \mathrm{AU}$ at $10^{7}$ years for the minimum-mass solar nebula model $\left(\Sigma_{1}=7 \mathrm{~g} / \mathrm{cm}^{2}\right.$ and $\left.q=3 / 2\right)$ as a function of the initial planetesimal mass. The symbols and lines are same as Fig. 8 ,

Fig. [12 - Same as Fig. 11, but for $\Sigma_{1}=70 \mathrm{~g} / \mathrm{cm}^{2}$.

Fig. 13 - Evolution of the embryo mass at $3.2 \mathrm{AU}$, neglecting fragmentation. The dashed lines indicate $M_{\mathrm{n}}$ (Eq. A3; black) and $M_{\mathrm{e}}$ (Eq. A4, gray). 


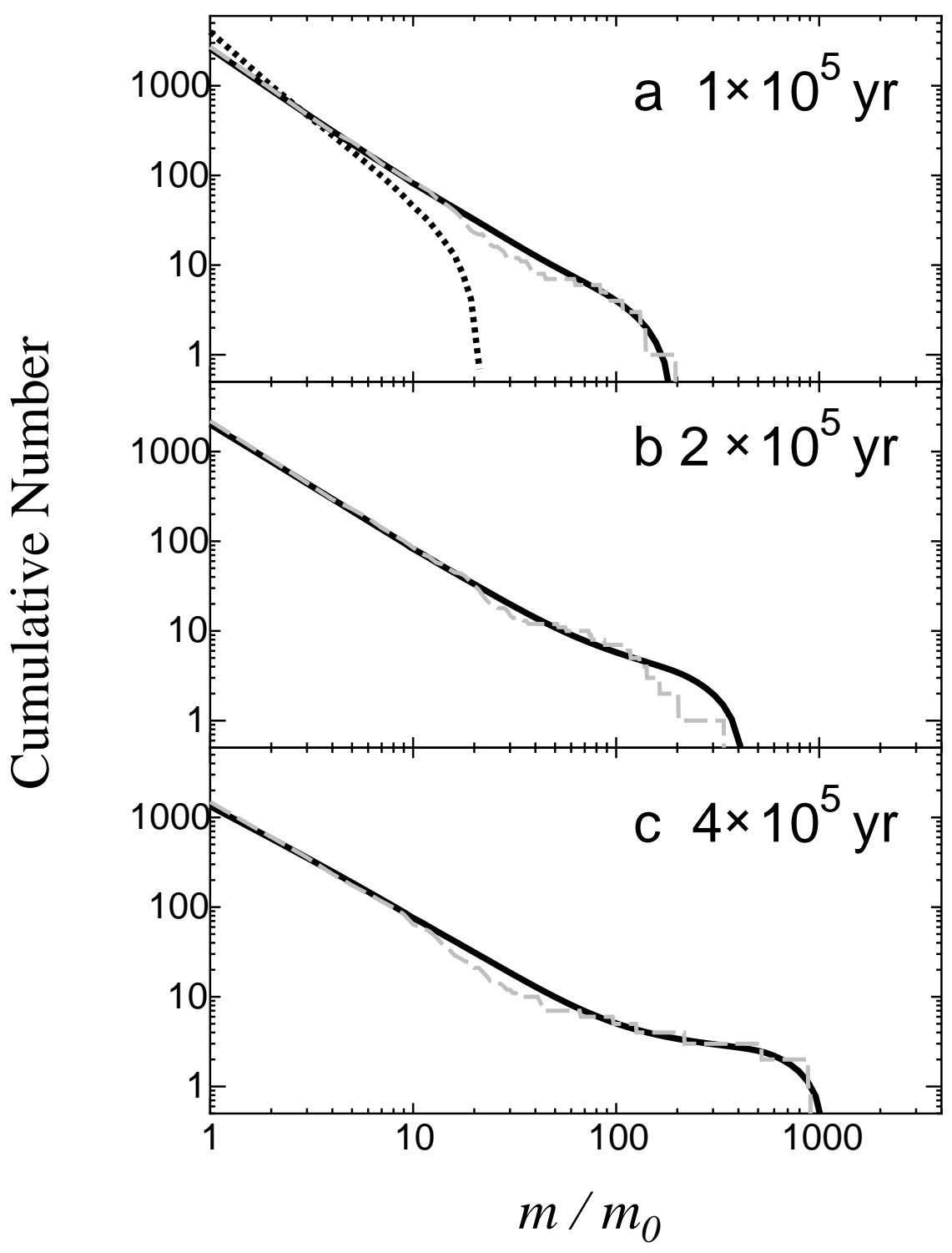

Fig. 1.- 


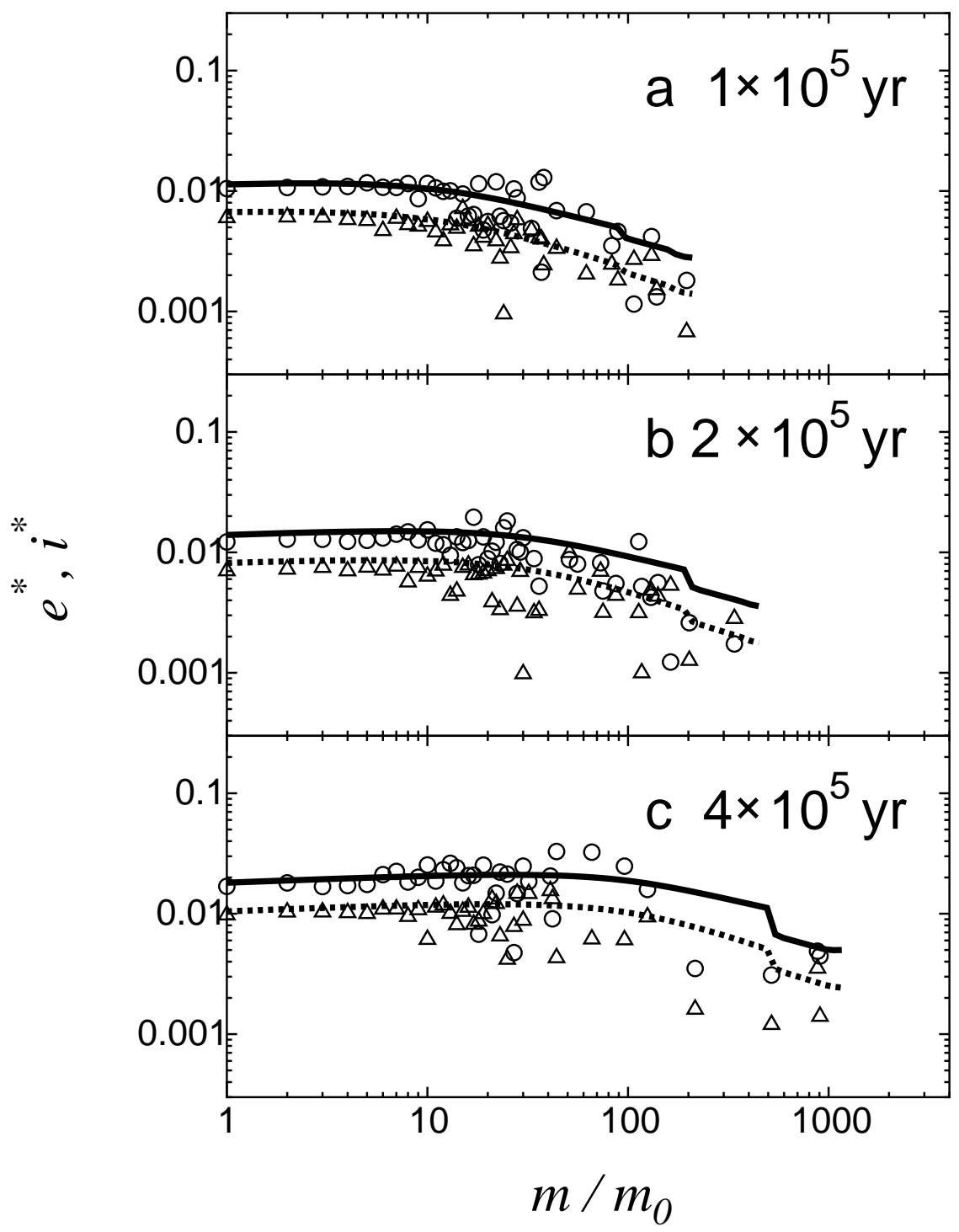

Fig. 2.- 


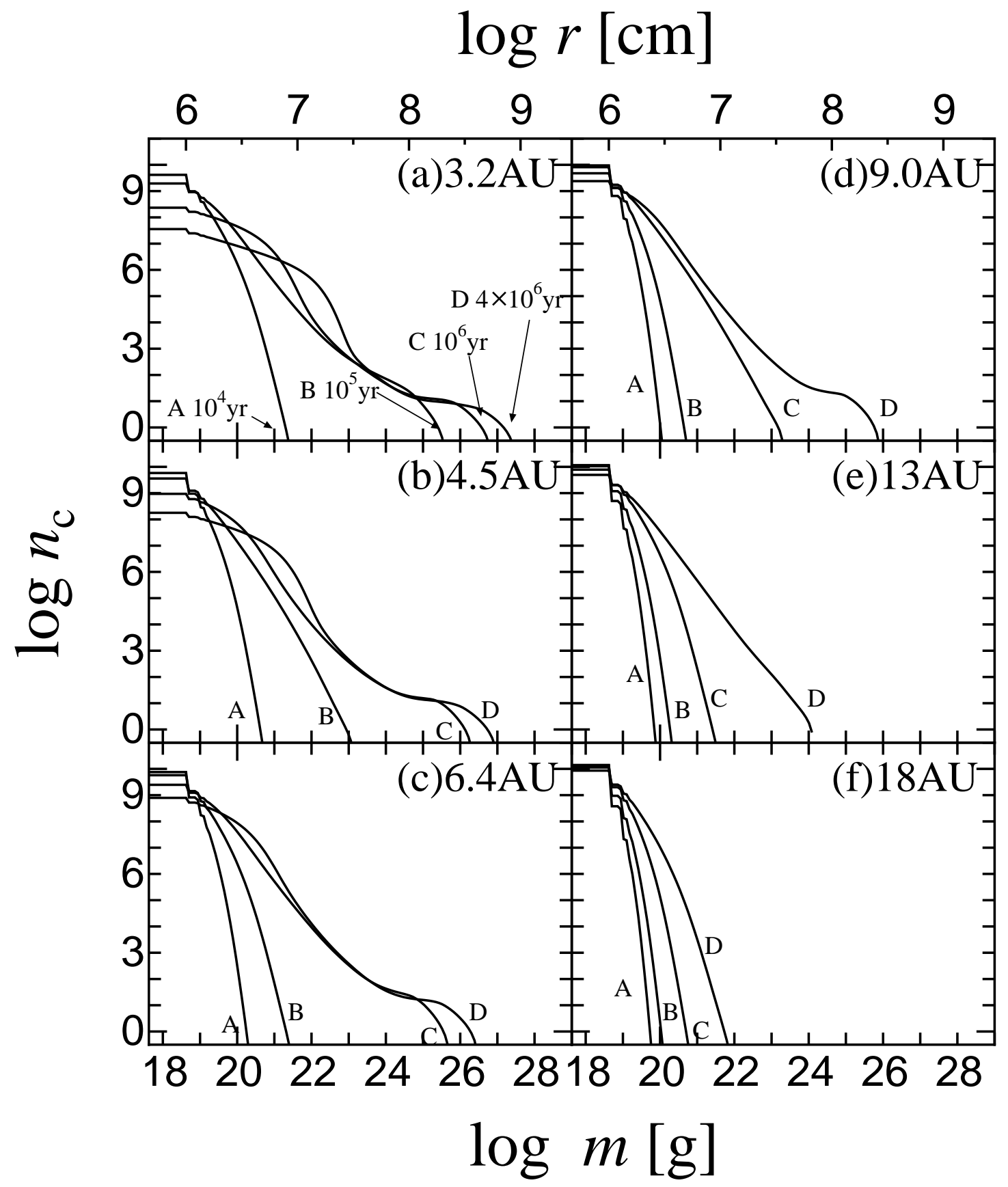

Fig. 3.- 


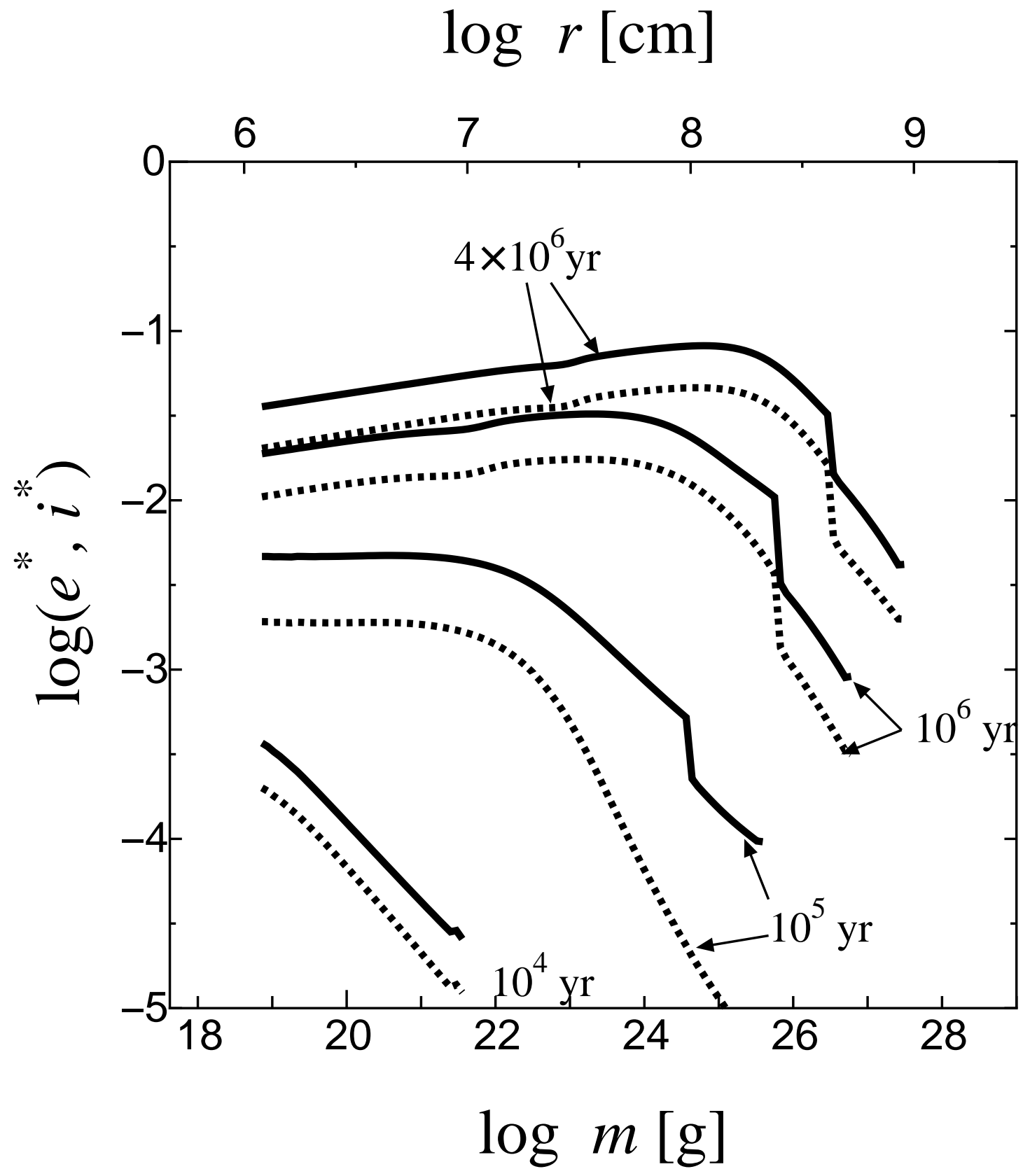

Fig. 4.- 


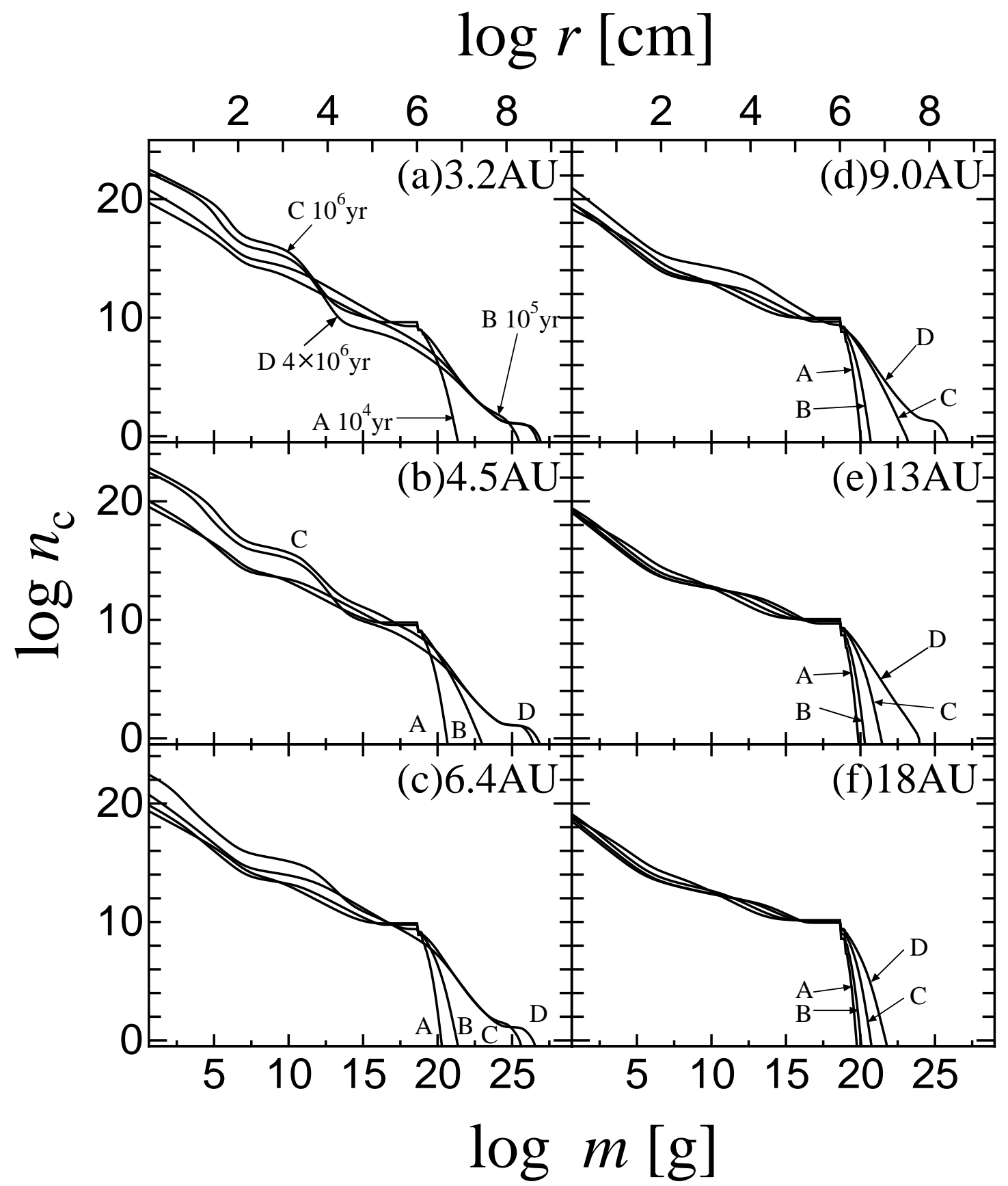

Fig. 5.- 


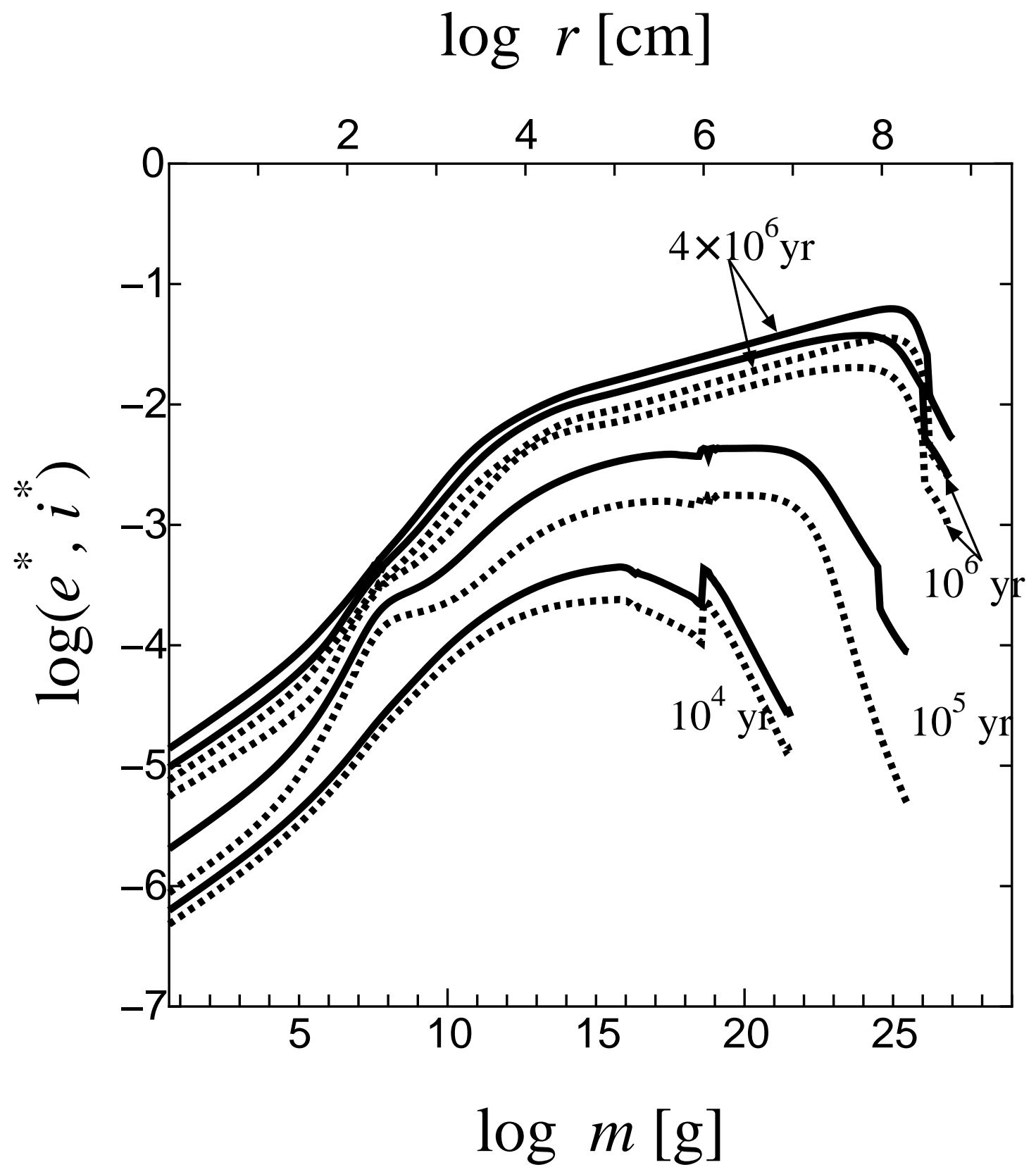

Fig. 6.- 


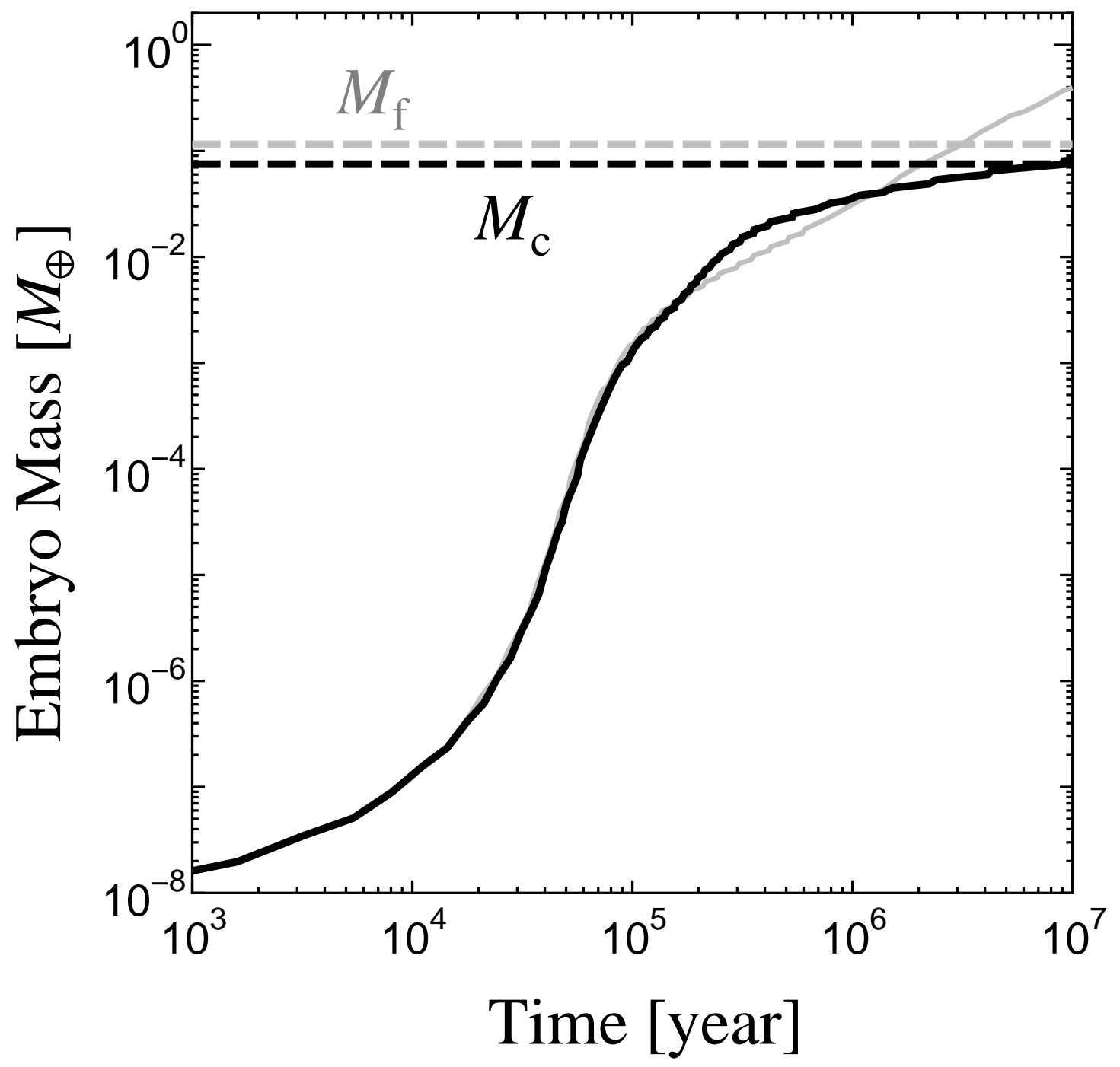

Fig. 7.- 


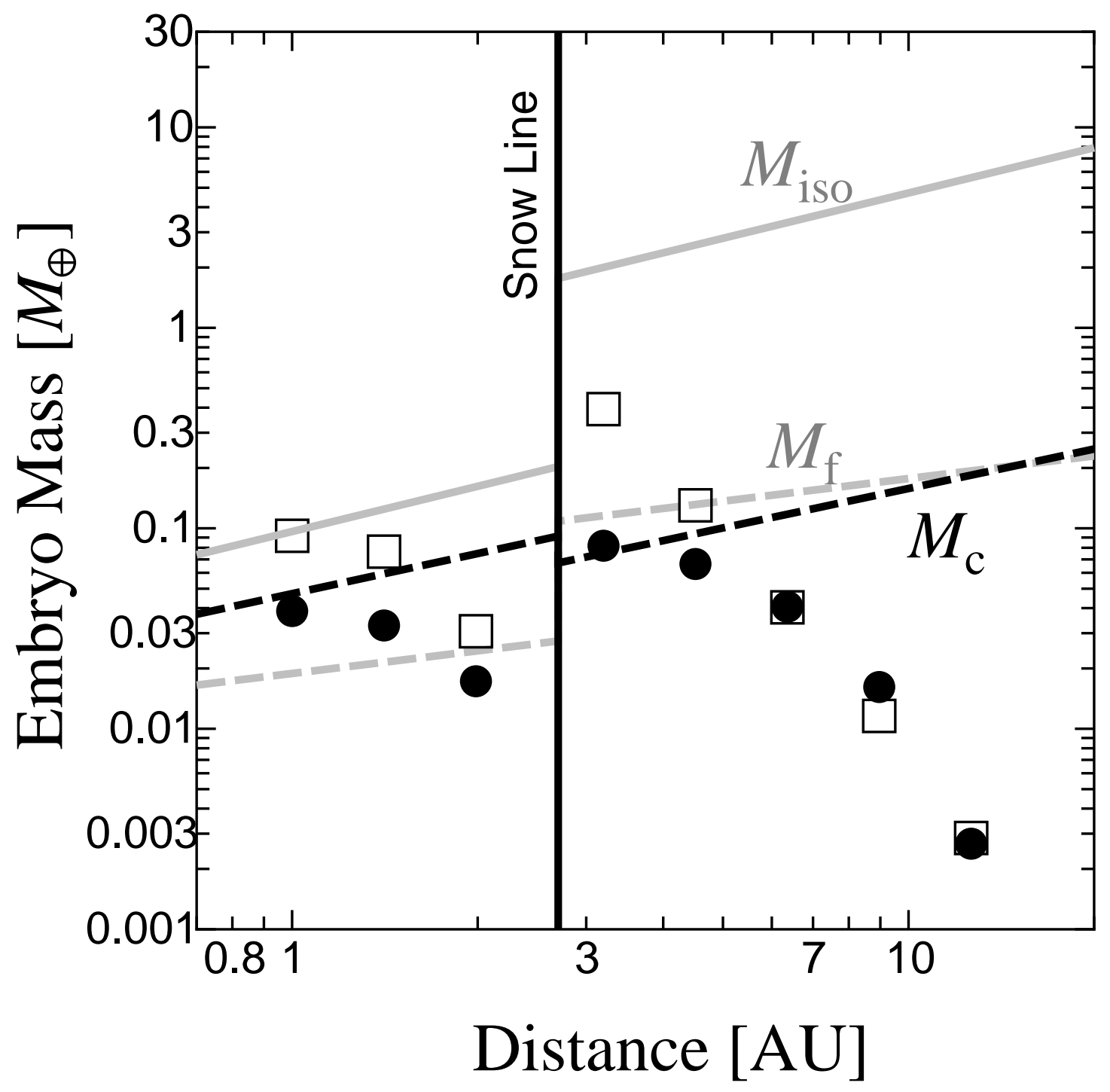

Fig. 8.- 


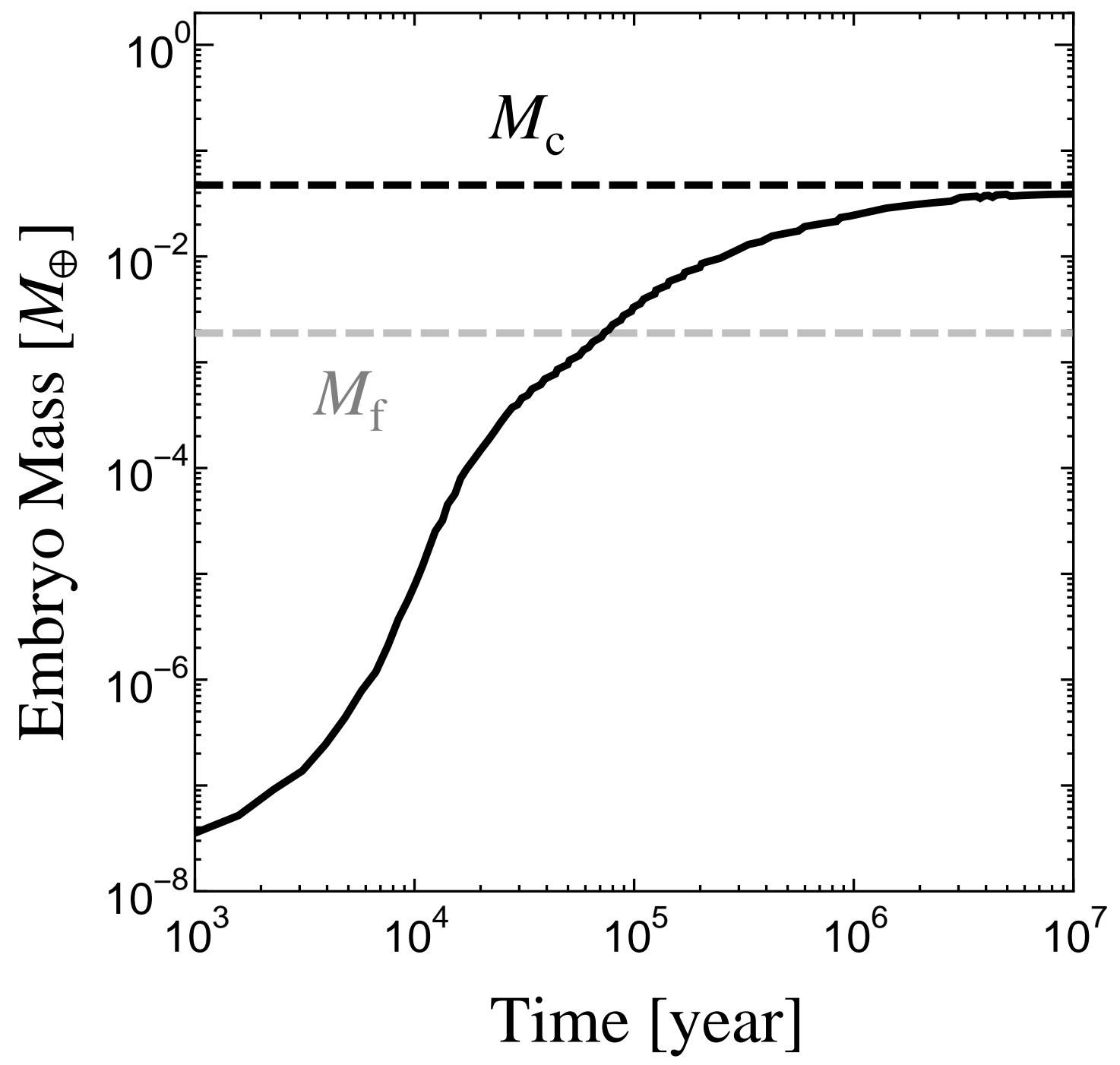

Fig. 9.- 


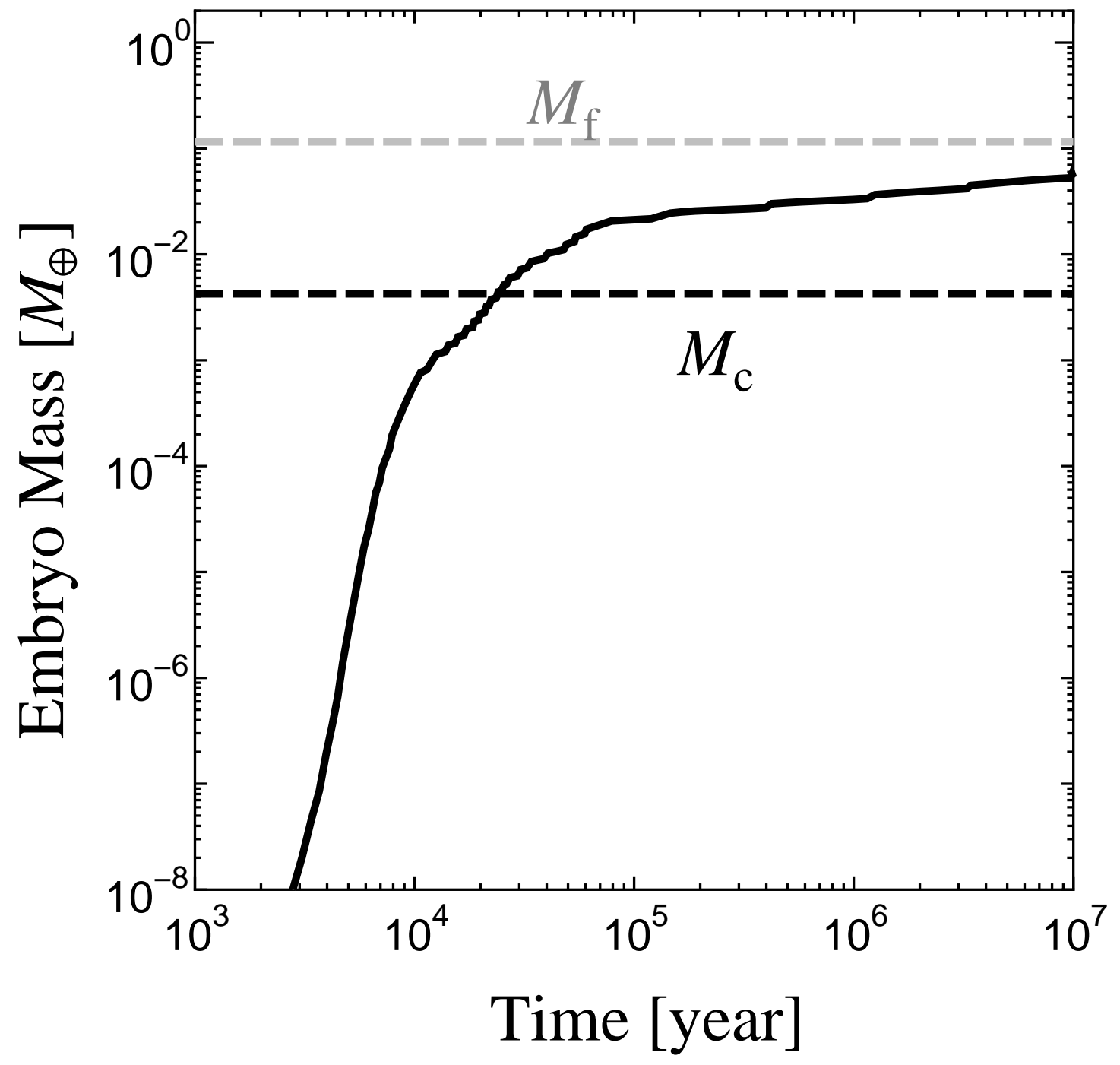

Fig. 10.- 


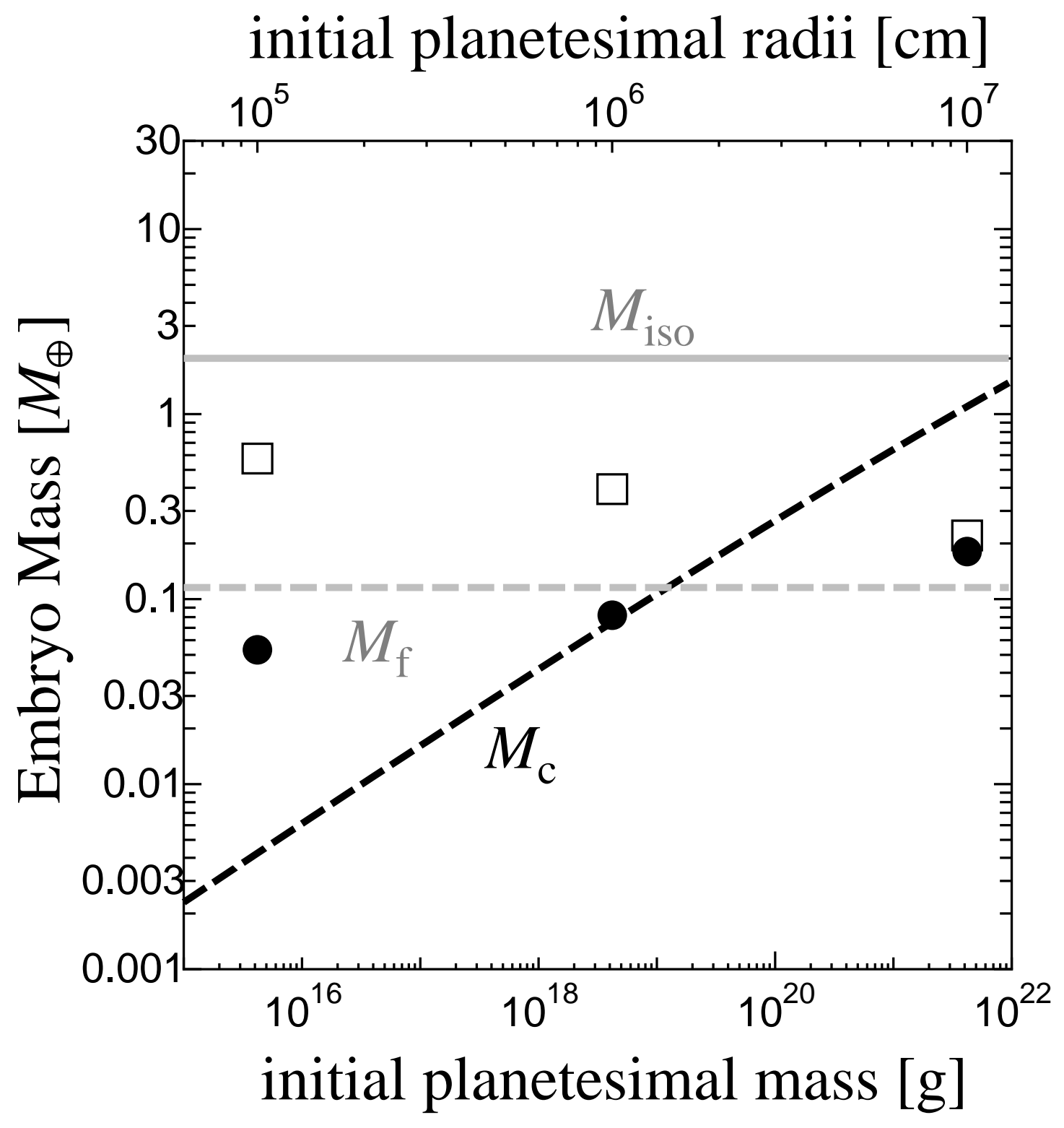

Fig. 11.- 


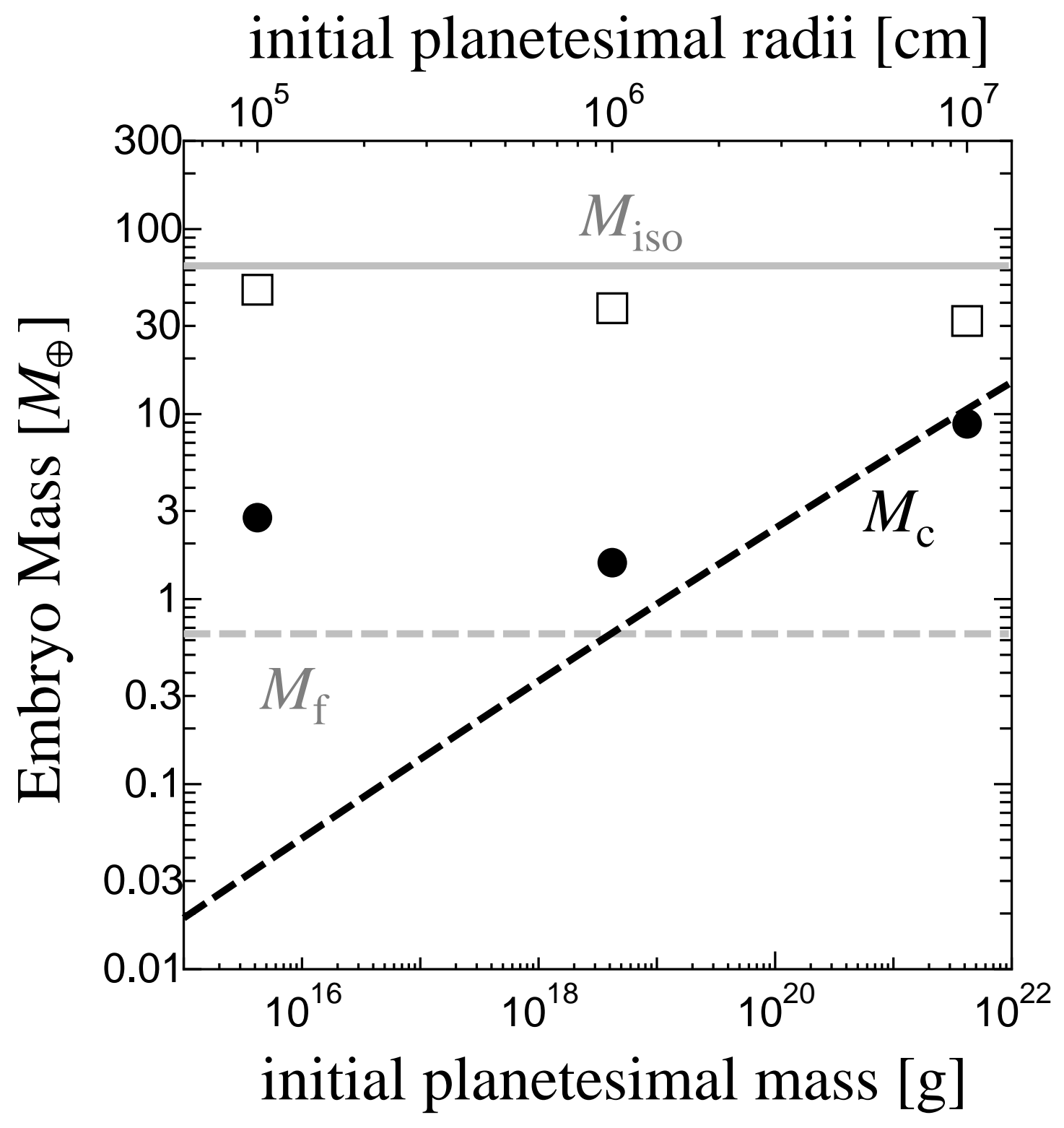

Fig. 12.- 


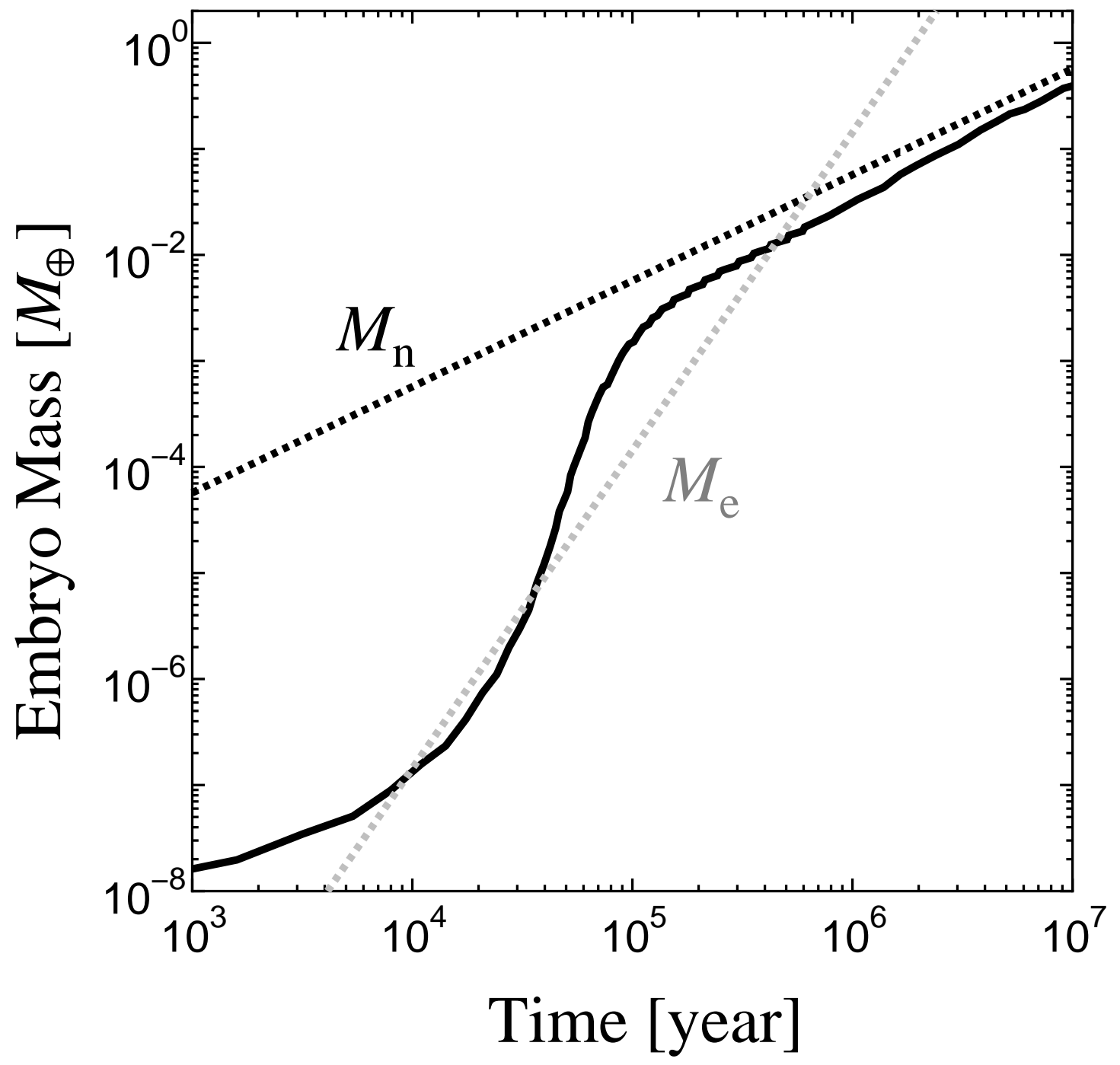

Fig. 13.- 